\title{
La dinámica de las redes de amistad. La elección de amigos en el programa Erasmus. ${ }^{1}$
}

\author{
Ainhoa de Federico de la Rúa ${ }^{2}$ \\ Marie Curie Fellow en el ICS-Universidad de Groningen (Holanda). \\ Universidad de Lille, Ifresi-Clersé-Cnrs (Francia) \\ Universidad Pública de Navarra (España)
}

\section{Resumen}

Examinamos la emergencia y evolución de una red total de amistad de estudiantes europeos de intercambio a partir de hipótesis teóricas sobre la amistad tomadas de la Psicología social y la sociología.

Esta red de amistad ha evolucionado a partir de una situación en que prácticamente nadie conocía a los demás.

Estudiamos los factores potencialmente influyentes en el desarrollo de la red como la similitud (e.g. nacionalidad, sexo), las oportunidades de interacción (e.g. proximidad de lugar de residencia, estudios), los costes de la relación (e.g. nivel hablado de los distintos idiomas), y también los efectos estructurales como la reciprocidad, la transitividad, los niveles de actividad sociable y la popularidad. Como los datos fueron recogidos a partir de redes personales, es posible también considerar las alternativas fuera de la propia red. Para modelar la evolución de la red utilizamos modelos orientados hacia el actor implementados en SIENA.

Palabras clave: Evolución de redes sociales, amistad, estudiantes, intercambios europeos.

\footnotetext{
${ }^{1}$ Agradezco encarecidamente a Tom Snijders su ayuda para comprender y aprender a utilizar SIENA, así como sus buenas ideas sobre los análisis. También a Marko Pahor su ayuda para hacer los estupendos gráficos con Pajek.

${ }^{2}$ Enviad la correspondencia sobre este artículo a Ainhoa de Federico: federico@univ-lille1.fr
} 


\section{Introducción}

Desde mediados de los años 80, la Unión Europea ha invertido enormes cantidades de dinero 3 para fomentar la movilidad de los estudiantes con la idea enfatizada explícitamente en su discurso oficial ${ }^{4}$, de que los estudiantes europeos en intercambio (entendidos como vectores culturales) y la experiencia intercultural que provocan y en que participan, pueden ser un poderoso catalizador de la formación de la conciencia o identidad europea. En el discurso de la Unión Europea a propósito de los intercambios de estudiantes de algún modo está implícita la hipótesis de la Psicología social de que el contacto y la interacción promueven la solidaridad y la apreciación mutua en su afán por "reforzar el espíritu de ciudadanía europea" y "reforzar el entendimiento entre los pueblos de Europa". A partir de esta idea los esfuerzos han sido enormes y fructuosos: desde su implantación en 1987, tan sólo gracias al programa Erasmus (existen otros muchos) se estima que aproximadamente 1,5 millones de estudiantes universitarios han disfrutado de una estancia de 3 a 12 meses en otro país europeo. Se trata de una cifra importante si se compara con los 5 millones de europeos que viven de manera más o menos estable en otro estado miembro de la UE.

Sin embargo, las investigaciones llevadas a cabo sobre los estudiantes participantes de estos intercambios ${ }^{5}$ son decepcionantes, los impactos de la estancia en el extranjero sobre la apreciación del país de acogida son bastante limitados y no siempre positivos (Selltiz y Cook 1962, Shield 1962, Stroeber et al 1988, Opper et al 1990). En línea con ello, según Pettigrew, los estudiantes participantes en intercambios constituyen la mayor excepción, si no la única, a la hipótesis de que el contacto aumenta los sentimientos positivos. Incluso, lo decía ya Karl Deutsch (1952), las experiencias de estudios en el extranjero pueden conducir a reacciones negativas respecto al país de acogida. En el caso de los estudiantes en

\footnotetext{
${ }^{3}$ Por ejemplo, 840 Millones de euros sólo para el programa SOCRATES de 1990 a 1995.

${ }^{4}$ Sobre la importancia concedida por la UE a los programas de intercambio de estudiantes europeos y la evolución de su discurso a propósito de la importancia del desarrollo de la conciencia europea mediante éstos ver Ruiz-Gelices et al. (2000).

${ }^{5}$ Ver la serie de investigaciones llevadas a cabo por el equipo de la Universidad de Kassel bajo la dirección de Ulrich Teichler publicados en los Erasmus Monographs.
} 
intercambios Europeos, el poco cambio en las actitudes hacia los países de acogida parece tanto más sorprendente dado que las condiciones identificadas como favorables por la psicología social para la reducción de prejuicios (apoyo social e institucional, potencial de conocimiento personal, igualdad de estatus, posibilidad de interacción cooperativa, (Stroeber et al 1988 Brewer y Gaertner 2001)) a priori están presentes.

Sin embargo, la tan sorprendente excepción a la hipótesis del contacto en las evaluaciones de los programas europeos, no resulta tal cuando, mirando con detalle, nos damos cuenta de que dichos estudios dejan de lado examinar... icon quienes se relacionan los estudiantes! Tales contactos reales son las fuentes potenciales de influencia y emergencia de solidaridad.

La investigación de la que se deriva este artículo estudia ambos aspectos de las relaciones de amistad creadas por los estudiantes en programas de intercambio europeos: 1) La selección: ¿Cómo y con quién crean lazos de amistad? 2) La influencia: ¿Cuáles son los efectos de la amistad en las percepciones de los otros y en la identificación con Europa? ${ }^{6}$. Este artículo presenta la emergencia de las relaciones de amistad, más concretamente entre estudiantes Erasmus. Comprender cómo y con quién interactúan los estudiantes de intercambio ya es un primer paso para comprender qué efectos se pueden esperar de tales contactos.

\section{Hacerse amigos}

La cuestión del contacto y la interacción no sólo trata de relaciones de amistad. En efecto, en la vida cotidiana existen todo tipo de contactos con personas ocupando roles variados más o menos institucionales y más o menos impersonales (en el caso de los estudiantes por ejemplo los profesores, los administrativos, el casero, el panadero...) que pueden ser agradables o desagradables, eficaces o no, y contribuir a formar opiniones y predisposiciones a propósito de un país. Sin embargo, es indudable que las relaciones de amistad están fundadas directamente en la solidaridad y la confianza, y por lo tanto al mismo tiempo las requieren y las construyen.

\footnotetext{
${ }^{6}$ Sobre la influencia de la amistad en la percepción de los otros ver de Federico (2002b).
} 
Partimos del supuesto de que si hay un tipo de contacto o interacción que pueda conducir a extender el entendimiento, la confianza y la solidaridad de forma inductiva, esto es, de casos particulares a la generalidad, se trata de la relación de amistad ${ }^{7}$.

¿Pero qué son las relaciones de amistad? Paine (1969) y Allan $(1979,1989)$ las definen como relaciones personales, voluntarias y principalmente afectivas (aunque también puedan ser instrumentales). En ellas se pueden distinguir niveles de intensidad (Allan 1979, Fischer 1982a, Kurtz 1970): desde los simples conocidos "amistosos" a los "amigos del alma" existe una gradación cuya nomenclatura difusa oculta casi tanto como revela.

¿Cómo se forman las amistades? Primero, a la gente le suele gustar tener amigos y es lógico: los amigos son una fuente importante de identidad, son los que saben "quiénes somos realmente" y nos aceptan tal cual (Allan 1989, Bidart 1991, 1993). También nos hacen la vida más agradable y más fácil: comparten nuestros intereses e ideas (Lazarsfeld y Merton 1954, Bidart 1991), nos ayudan tanto con las cuestiones de la vida cotidiana (Wellman et al 1988, Wellman y Wortley 1990), como con los momentos de grandes crisis existenciales (Ferrand 1993). Segundo, las amistades, por muy mágicas, específicas, particulares y personales que parezcan, se forman en contextos sociales y están sujetas a normas y modelos sociales (Ferrand 1993). Como mínimo, es necesario estar en el mismo sitio al mismo tiempo, haciendo algo que permita una interacción mínima en un momento dado, para poder conocer a alguien, descubrir que nos cae bien y hacernos amigos. Por lo tanto, para la formación de relaciones de amistad existe una estructura de oportunidades ligada a aspectos puramente geográficos (dónde pasamos nuestro tiempo), pero también de interdependencia funcional en focos de actividad (qué hacemos y qué interacciones permite la actividad) (Feld 1997, Flap y Volker 2002). A partir del momento en que existe la posibilidad de interacción, en general parece que las personas prefieren hacerse amigos de personas similares a ellos, las hipótesis adelantadas sobre por qué ocurre esto son que las relaciones

\footnotetext{
${ }^{7}$ Ver de Federico 2001 y 2002b para una argumentación más extensa sobre la importancia de la amistad como catalizadora de solidaridades abstractas más allá de la propia relación interpersonal.
} 
positivas con personas de iguales valores, actitudes y características sociales reducen la disonancia cognitiva (Heider en Perlman y Fehr 1986) y porque confirman la idea de uno mismo, procuran más apoyo emocional y menos críticas (Suitor y Keeton 1997). Sin embargo hay también argumentos para defender la complementariedad entre los amigos. Breiger y Roberts (1998, p. 241) resumiendo a Durkheim dicen:

\begin{abstract}
"¿Se basa entonces la amistad en la similitud o en la diferencia entre los amigos? Esta cuestión es expuesta en el primer capítulo de la obra de 1893 de Durkheim La división social del trabajo. Después de revisar diversas posiciones tomadas por pensadores clásicos (Aristóteles, Eurípides, Heráclito) y por autores modernos en esta cuestión, Durkheim concluye que "buscamos en nuestros amigos las cualidades que nos faltan, puesto que al reunirnos con ellos, participamos en cierta medida de su naturaleza y nos sentimos menos incompletos" (1893, pp.55-6). Es esta división del trabajo la que determina la relación de amistad. De hecho la verdadera función de la división del trabajo es crear en dos o más personas un sentimiento de solidaridad." (traducción propia)
\end{abstract}

Bajo esta perspectiva, serían las diferencias y no las similitudes las que constituirían la base deseable para desarrollar las relaciones de amistad.

Además, a menudo las relaciones se establecen a partir de otras relaciones (Hallinan 1979, Ferrand y Mounier 1999): nuestras redes personales nos permiten tener acceso a nuevos contactos (e.g. eventualmente los amigos de mis amigos pueden convertirse en mis amigos (Granovetter 1973, Degenne y Forsé 1994), al mismo tiempo que los limitan (e.g. Ios enemigos de mis amigos no pueden ser mis amigos).

Hasta aquí hemos mencionado factores clásicos en las teorías de la amistad: Partiendo de un supuesto gusto o preferencia por tener amigos, hemos mencionado la importancia de la estructura de oportunidades de contacto, la supuesta preferencia por la similitud, y el acceso condicionado a nuevas relaciones a partir de las anteriores. Además nos parece importante insistir en el papel de los roles y en el de las redes sociales en tanto que controladoras (o no) de las normas. Los roles ocupados por los actores son importantes en tanto que estructuran los focos de actividad o los contextos 
de interacción, así como las normas y preferencias que se aplican a éstos y por lo tanto las similitudes relevantes en estos contextos. Por otra parte, las redes son las que pueden controlar de manera efectiva las normas (Ilamadas en este caso por Ferrand (1997) "normas prácticas") y por lo tanto permiten o imposibilitan ciertos amigos potenciales. El control efectivo ejercido por las redes, o la idea que los actores se hacen de cómo sería este control puede también influir la elección de los amigos.

Ésta última observación es importante porque los individuos que estudiamos ocupan dos roles estructuradores: el de estudiantes y el de extranjeros. Particularmente el segundo puede tener un papel específico en la formación de amistad en el contexto en que la analizamos. Según Simmel (1908) un extranjero es a la vez un elemento interior y exterior al grupo. No se trata de alguien que está de paso, sino un individuo potencialmente móvil. Su posición implica al mismo tiempo la exterioridad y la oposición al grupo que lo acoge y representa una síntesis de la proximidad y de la distancia física, social y simbólica. Ser extranjero se convierte en una característica esencial de definición de un individuo y de una categoría de personas. Finalmente percibimos al extranjero, no en tanto que individuo, sino en tanto que extranjero de un tipo particular. Además podríamos argüir que el extranjero, tiene una relación especial con las normas del grupo que lo acoge. Las reglas no se aplican a él de la misma manera que al grupo por dos motivos: 1) Hasta cierto punto se tolera que éste cuente con otras normas de comportamiento. 2) su posición alejada de sus relaciones personales primordiales más importantes para la confirmación de su comportamiento hacen que éste esté menos sujeto a un control efectivo a ciertas normas prácticas.

En este punto conviene también hacer alusión al hecho de que, aunque en nuestras sociedades occidentales la amistad es una relación personal, privada, voluntaria y no ritualizada (Paine 1969), su definición, contenido y expectativas puede diferir según los países. Baste constatar la diferencia de términos que se emplean para aludir a ella en distintos países: en francés se distingue entre ami y copain (siendo copain considerada una relación más ligera). En inglés británico entre friend y mate, mientras que los 
estadounidenses utilizan tan solo friend o eventualmente friendly relation (y de hecho Fischer (1982b) ha encontrado diferencias sistemáticas entre las definiciones de estadounidenses y británicos siendo estos últimos menos propensos a calificar a sus relaciones de amigos). En castellano, sin embargo sólo utilizamos "amigo" para referirnos a ambos matices, y en griego (moderno), el término para designar a los amigos es incluso más amplio: la palabra $\varphi \imath \lambda o s$ engloba también al novio o la novia. Podemos esperar entonces que diferencias respecto a cómo se entiende la amistad según los países muestren sus efectos en los modelos de sociabilidad de los estudiantes.

\section{El caso: estudiantes Erasmus en Lille.}

Para examinar la dinámica de las relaciones de amistad estudiamos 103 participantes del programa Erasmus venidos de 10 países diferentes ${ }^{8}$ a la Universidad de Lille en octubre de 1995. Realizamos dos medidas de sus redes de amistad: La primera tuvo lugar en febrero de 1996, justo antes de que los primeros estudiantes volvieran a sus países de origen. Administramos un cuestionario estandarizado con un módulo para describir las relaciones de amistad. El generador de nombres utilizado fue amplio "¿Podrías mencionar el nombre de tus amigos" durante tu estancia Erasmus?" Esta pregunta pretendía capturar todo tipo de amigos de intensidades variadas. Además, identificamos hasta 4 mejores amigos. Preguntamos también por los amigos que ya conocían antes del periodo de intercambio, obligatoriamente pocos, ya que los estudiantes venían de procedencias muy diversas. Finalmente pedimos una descripción bastante detallada de las características de los amigos.

La segunda medida tuvo lugar en junio de 1997, una vez que ya había pasado un año desde que los últimos estudiantes volvieron a sus casas, mediante cuestionarios postales. El generador de nombres utilizado fue el mismo "¿Podrías mencionar el nombre de tus amigos durante tu estancia

\footnotetext{
8 Alemania, Austria, Bélgica, España, Gran Bretaña, Grecia, Holanda, Italia, Portugal y Suecia. Ver la tabla de distribución de las nacionalidades en la población y la muestra en el anexo.

${ }^{9}$ La pregunta originalmente en francés preguntaba por los "amis et copains". No existe en castellano una palabra equivalente a "copain", compañero tiene connotaciones diferentes.
} 
Erasmus?". Además de las preguntas de la primera medida, pedimos que nos indicaran con qué frecuencia tuvieron contacto con estos por e-mail, carta, teléfono o cara a cara.

Tabla 1. Ficha de las medidas

\begin{tabular}{|c|c|c|c|}
\hline Periodo & Octubre 1995 & Febrero 1996 & Junio 1997 \\
\hline Punto del tiempo & TO & $\mathrm{T} 1=\mathrm{T} 0+4$ meses & $\mathrm{T} 2=\mathrm{T} 1+16$ meses \\
\hline Respuesta & $77 \%$ & $77 \%$ & $29 \%$ \\
\hline \multirow[t]{3}{*}{ Información disponible } & Conocidos & -------------------- & 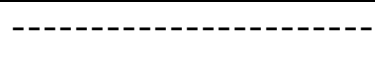 \\
\hline & Amigos & Amigos & Amigos \\
\hline & ------------ & Mejores amigos & Amigos contactados \\
\hline
\end{tabular}

No había arreglos institucionales que favorecieran particularmente la interacción exclusiva entre estudiantes Erasmus, y de hecho utilizamos un protocolo de redes personales, sin embargo los resultados mostraron que el $80 \%$ de los amigos de los estudiantes Erasmus estudiados eran otros estudiantes Erasmus. Sólo el $17 \%$ de los amigos eran estudiantes locales franceses y el $3 \%$ restante eran estudiantes de nacionalidades no europeas. La auto selección es impresionante si se considera que los estudiantes Erasmus constituían solamente el $1,7 \%$ de la población estudiante de la universidad y que estaban dispersos en estudios y lugares de residencia diferentes en que eran minorías. Así pues, la primera constatación es que entrar en el rol Simeliano de extranjero ${ }^{10}$ tuvo el mayor impacto en las formas de sociabilidad de los estudiantes Erasmus. Una similitud basada en la diferencia, la de ser extranjeros, es la más estructuradora. Los estudiantes Erasmus pueden ser culturalmente diferentes y socioeconómicamente diferentes (aunque se podría asumir probablemente sin equivocarse demasiado que sus orígenes socioeconómicos son parecidos, dada la escasez de la beca Erasmus), sin embargo el hecho de que ocupen la misma posición, que se hayan convertido en el mismo tipo de extranjeros, hace que ésta sea su característica principal y por lo tanto la más estructuradora. 
Así pues, constatamos que los estudios en psicología social sobre la hipótesis del contacto en el caso de los estudiantes de intercambio postulan el contacto donde apenas existe y dejan de lado estudiar el contacto allí donde lo hay, es decir entre los estudiantes de intercambio de diferentes países.

Dada la constitución de un círculo social de estudiantes Erasmus, tiene sentido desde un punto de vista sustantivo reconstruir la red total de relaciones entre estudiantes Erasmus. A su vez, esto permite examinar datos de mayor riqueza estructural. A partir de este momento, la cuestión a estudiar es, dada la situación inicial (TO), ¿cómo evoluciona la red de amistad de T0 a T1 y de T1 a T2? Por cuestiones de extensión en este artículo sólo consideramos la evolución de la red de T0 a T1.

\section{Modelos estadísticos para redes totales}

Para estudiar la emergencia de la red total de amistad entre estudiantes Erasmus necesitamos un modelo estadístico específico capaz de estudiar los efectos estructurales, preferentemente de forma longitudinal, ya que los datos están disponibles. También necesitamos que este modelo sea capaz de tener en cuenta variables sociológicas, exógenas a la red e importantes para el desarrollo de la red de amistad. Dada la escasez de literatura en español sobre este tema, se presenta en esta sección una breve historia de los modelos estadísticos para redes totales que explican la estructura de la red a partir de procesos locales. A su vez, esto permitirá justificar la elección de SIENA para el presente análisis.

El primer modelo estadístico para redes totales, desarrollado por Holland y Leinhardt (1981), es conocido bajo el nombre de modelo p1 (primer modelo de probabilidad). Las técnicas de estimación loglineares del modelo $p 1$ fueron desarrolladas por Wasserman et al (Wasserman et Faust, 1994). Este modelo incluía en sus especificaciones efectos individuales y efectos diádicos, pero no efectos triádicos. Los efectos individuales que permitía tratar eran la popularidad (relaciones recibidas o grado de entrada) y la

\footnotetext{
10 Ver de Federico 1997 y 1998 para una descripción más detallada de la situación relacional del los estudiantes Erasmus en tanto que extranjeros.
} 
actividad (relaciones emitidas o grado de salida) de un actor, pero no permitía tener en cuenta efectos debidos a variables exógenas de carácter no relacional, como el sexo, la edad, el estatus, u otras características individuales que pueden ser cruciales para el desarrollo de la estructura de una red total. En cuanto a las variables estructurales, sólo permitía examinar la reciprocidad (probabilidad de que si $i$ emite una relación hacia $j$, $j$ emita también una relación hacia $i$ ), lo que se queda en el nivel diádico de la estructura. Este modelo puede ser entendido como un modelo de regresión logística bivariable para díadas. Éste postula la independencia entre díadas, lo que impide un tratamiento triádico y por lo tanto plenamente estructural. Sin embargo su creación fue un punto de partida importante para la modelización estadística de las redes sociales.

Algunas de las limitaciones del modelo $p 1$ fueron superadas por el modelo p2 desarrollado por van Duijn (1995, Lazega y van Duijn 1997). El modelo p2 puede ser considerado como una versión con efectos aleatorios o como una versión multi-nivel del modelo $p 1$. Los métodos de estimación para el modelo p2 son bastante complejos. Actualmente van Duijn y Ziljstra siguen mejorándolos. La aportación principal del modelo $p 2$ es que permite la inclusión y el tratamiento, en tanto que variables independientes dentro del modelo, de variables individuales exógenas (edad, sexo, o cualquier otra característica de los actores) y de variables diádicas (como las similitudes, flujos, frecuencias, contenidos, o cualquier otra característica de las relaciones). También permite estudiar los efectos de interacción entre las variables explicativas y la reciprocidad. Para un análisis sociológico, el modelo $p 2$ es ya mucho más interesante que el modelo $p 1$ puesto que permite incorporar variables explicativas clásicas de la sociología (ver los ejemplos de Lazega 2001). Sin embargo, desde un punto de vista estructural, aunque aumenta las posibilidades de análisis a nivel diádico mediante la incorporación de variables diádicas y el estudio de las interacciones entre las variables explicativas y la reciprocidad, el modelo $p 2$ sigue siendo limitado, ya que no incorpora efectos triádicos. 
Figura 1. Las clases isomórficas de tríadas según el esquema MAN de Holland y Leinhard

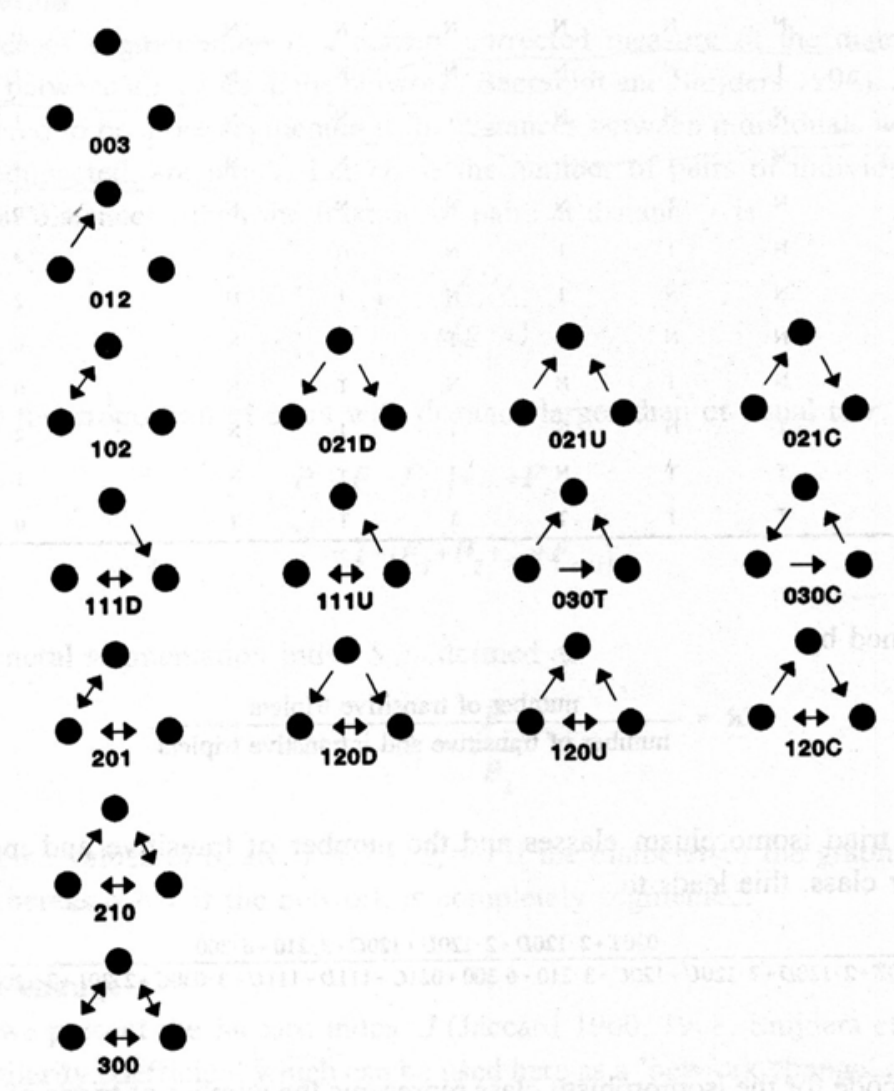

En 1996 Wasserman y Pattison propusieron el modelo $p^{*}$ generalizando la distribución de grafos de Markov propuesta por Frank y Strauss (1986). Este modelo propone una formulación marco extremamente general y capaz de representar cualquier distribución de probabilidades de un grafo dirigido. Esto permite una gran versatilidad y la incorporación de efectos triádicos. Su ventaja es de algún modo su inconveniente: esta formulación es tan general que es necesario encontrar las diferentes formulaciones específicas para "rellenar" la fórmula y volverla operativa. Desde su aparición en 1996 diversos investigadores se han consagrado a la elaboración de este modelo (Wasserman, Pattison, Robins y otros). Por otra parte, el procedimiento de estimación de parámetros es provisional, hasta ahora (tal como está implementado en el programa Prepstar), se venía utilizando un procedimiento de pseudo-probabilidad que reduce la estimación a un procedimiento de regresión logística. Sin embargo las propiedades 
estadísticas de este procedimiento de estimación todavía son desconocidos y por lo tanto es mejor tomar los resultados con cautela. Muy recientemente Snijders (2002) ha propuesto un procedimiento más fiable de estimación basado en cadenas de Markov Monte Carlo, disponible en también en el programa StOCNET desde julio 2002.

\section{Figura 2 Tripletes transitivos e intransitivos}

Triplete (ijk) en estado transitivo
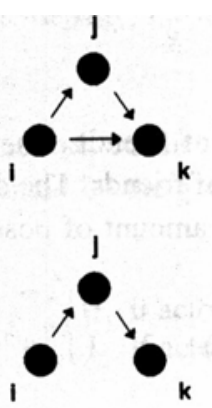

Triplete $(i k j)$ en estado transitivo

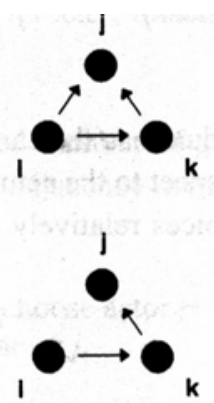

Triplete ( $i j k)$ en estado intransitivo Triplete $(i k j)$ en estado intransitivo

Una vez dicho esto, el modelo $p^{*}$, además de efectos diádicos, ha posibilitado todo tipo de análisis triádicos mediante el recuento de tipos de tripletes. Una triada representa las relaciones recíprocas (o no) entre 3 actores (ver la Figura 1 basada en Holland y Leinhard 1976). Se llama triplete a una tríada que tiene en cuenta el punto de vista del actor (ego). Un recuento de tripletes representa simplemente el número de veces que se encuentra un triplete particular en la red. Por razones teóricas son particularmente interesantes dos tipos de triplete, los que se llaman triplete intransitivo y triplete transitivo (ver la Figura 2).

Al igual que el modelo $p 2$, el modelo $p^{*}$ permite la incorporación de variables explicativas individuales $y$, además, lo supera permitiendo estudiar tres efectos ligados a las variables individuales: la actividad, la popularidad, y la disimilitud (por ejemplo permite estudiar si los hombres son más activos que las mujeres, o menos populares, y si los actores prefieren entrar en relación con personas del mismo sexo o no). A menudo, cuando se utiliza el modelo $p^{*}$ se incorporan medidas estadísticas que representan la densidad, la reciprocidad, la actividad, la popularidad, las relaciones 
indirectas y la transitividad. El modelo $p^{*}$ permite también, al igual que $p 2$, estudiar efectos diádicos exógenos.

Hasta ahora los modelos presentados son estáticos, pero ya que las redes son estructuras que se desarrollan y cambian con el tiempo, es necesario comprender el desarrollo de las mismas, lo que pretendemos aquí. Para ello ha sido necesario desarrollar modelos que tengan en cuenta el paso del tiempo. Un procedimiento alternativo y más ventajoso ${ }^{11}$ que los modelos loglineares, es el uso de procesos de Markov para estudiar el desarrollo de las redes en el tiempo, tal como se ha propuesto en SIENA.

Cuando ambos modelos (SIENA y $p^{*}$ ) permitan tanto el modelado dinámico como el modelado de todos los efectos triádicos, su principal diferencia será que $p^{*}$ es un modelo orientado hacia la relación y SIENA un modelo orientado hacia el actor. El modelo más apropiado dependerá del grado de voluntariedad del tipo de relación en estudio: para relaciones voluntarias en que se puede considerar que los actores escogen a sus asociados, por ejemplo las relaciones de amistad, SIENA sería más apropiado, sin embargo, para relaciones en que el margen de libertad de los actores es limitado o nulo, como por ejemplo las relaciones de trabajo, $p^{*}$ se adaptaría mejor.

Tabla 2. Modelos estadísticos para redes sociales

\begin{tabular}{|c|l|c|c|c|}
\hline Modelo & Efectos & Díadas & Tríadas & Dinámica \\
\hline$p 1$ & Endógenos & Sí & No & No \\
\hline$p 2$ & Endógenos y exógenos & Sí & No & No \\
\hline$p^{*}$ & Endógenos y exógenos & Sí & Todos los tripletes & No $^{1}$ \\
\hline SIENA & Endógenos y exógenos & Sí & Efectos de cierre & Sí \\
\hline
\end{tabular}

1. Pattison, Robins y otros están trabajando en la versión dinámica de $p^{*}$.

2. Snijders, Huisman y otros están trabajando para incorporar más tripletes y otros efectos. Hay que tener en cuenta que para incorporar los tipos de tripletes en un modelo dinámico hay que tener hipótesis teóricas sobre las transiciones de un tipo de triplete a otro.

En nuestro caso, en que tratamos de relaciones de amistad, utilizamos SIENA. Dado lo novedoso del modelo, todavía en desarrollo, consideramos interesante presentarlo brevemente a continuación.

\footnotetext{
${ }^{11}$ Sobre las ventajas e inconvenientes de utilizar procesos de Markov en lugar de modelos loglineares, ver van de Bunt (1999, p. 31).
} 


\section{SIENA ${ }^{12}$}

Como ya hemos dicho, SIENA es un modelo estadístico para el análisis longitudinal de redes completas. La variable dependiente estudiada es el cambio de relaciones en la red total representada por uno o más grafos dirigidos entre un número fijo de actores $^{13}$. Las relaciones son definidas como dicotómicas (simplemente la presencia o la ausencia de determinado tipo de relación). El modelo adopta la teoría de la acción intencionada (purposive action) en que los actores escogen establecer, mantener o retirar relaciones para maximizar su utilidad. Las elecciones de los actores determinan la estructura de la red. El estado de la red en un momento determinado de la red permite y limita sus estados posteriores, así, la red es entendida como una estructura dinámica que permite y/o limita la propia evolución de la red.

El modelo permite el estudio de variables estructurales (reciprocidad, transitividad, cierre de la red...) endógenas a la dinámica de la red. También de variables exógenas, tanto las características de los actores (edad, sexo, tamaño del actor...), como las características de las relaciones (similitudes, frecuencias, intensidades, polivalencia, flujos...). Igualmente es posible estudiar algunas interacciones entre las variables endógenas y exógenas.

El modelo asume una serie de supuestos más o menos realistas, necesarios para el modelado matemático :

1) Los actores de la red pueden evaluar su posición en la red e intentar lograr una configuración de sus relaciones evaluada positivamente.

2) Dado el estado actual de la red, el modelo asume que todos los actores actúan de manera independiente (es decir sin coordinación).

\footnotetext{
12 Para una explicación más detallada sobre SIENA, ver de Federico (2002c) con una perspectiva más sociológica, o Snijders (2001) para una perspectiva más técnica. En castellano, ver la descripción presentada por Ainhoa de Federico en la Lista Redes. Se pueden consultar los archivos a partir de la página web de redes: http://revista-redes.rediris.es/webredes/ los mensajes son los no 000089 (19-900), no 000093 (25-9-00) y no 000102 (04-10-00) enviados por federico@univ-lille1.fr.

${ }^{13}$ En el futuro SIENA permitirá que desaparezcan o se incorporen actores nuevos a la red.
} 
3) Se supone que los actores tienen un conocimiento total del estado actual de la red.

4) Cada actor escoge las relaciones que emite.

5) Se supone que los cambios ocurren uno a uno y que todos los actores tienen la misma probabilidad de realizar un cambio.

6) Los cambios tienen lugar de forma no observada entre los momentos en que se mide la red.

7) Los estados futuros de la red son una función sólo del presente, se considera que el pasado relevante está contenido en el presente.

8) Los actores son "miopes", no tienen una estrategia a largo plazo, sino que buscan una satisfacción inmediata.

El realismo de tales supuestos es discutible y se adapta sin duda mejor, como ya se ha mencionado, a relaciones más bien voluntarias. Se podría argüir que:

1) La idea de evaluación e intento de mejorar la posición o de tener una posición satisfactoria implica una visión consecuencialista e instrumental de las acciones del sujeto. Como diría Boudon (1984), esto implica que el sujeto orienta su acción bajo la perspectiva de las consecuencias de lo que hace, que entre el conjunto de consecuencias que percibe, sólo presta atención a aquellas que le interesan y que el sujeto es capaz de determinar los costes y beneficios sus elecciones. Sin embargo sabemos por la literatura sociológica y antropológica sobre la amistad que, precisamente cuando los actores centran su atención en hacer el balance entre los costes y beneficios de una relación, nos hallamos frente a una situación de crisis (Bloch y Buisson 1991, Bidart 1991, Godbut y Charbonneau 1993). El estado normal de la relación de amistad es el de mutualidad altruista y no contabilizada.

Si admitimos que los actores sean estratégicos y evalúen su posición para tratar de mejorarla mediante cálculos racionales de costes y beneficios, 
entonces este supuesto se adaptaría mejor a tipos de relaciones más bien voluntarias sobre las que no pesan normas sociales muy restrictivas y en que los márgenes de libertad de los actores son relativamente grandes. Sin embargo, es posible también incluir variables para dar cuenta de los efectos de las normas y estimarlos en el modelo.

2) La independencia de la acción de los actores, la falta de coordinación, también se adapta mejor a relaciones voluntarias y sobre todo de carácter privado. Incluso en esos casos, en la literatura antropológica sobre la amistad encontramos fenómenos de exclusión colectivos en los grupos de amigos, por ejemplo en los modelos de amistad de las "cuadrillas" (Cucó 1995) en que la aceptación de nuevos miembros o su exclusión a menudo son fruto de decisiones fuertes y colectivas. Sin embargo, incluso si estos procesos existen en la realidad, para el tratamiento matemático es más sencillo hacer como si cada una de las decisiones de crear o eliminar relaciones tuviera lugar de forma independiente y en momentos diferentes, que pueden estar muy cercanos en el tiempo. Tal tratamiento es aceptable puesto que se puede considerar que antes o después una decisión colectiva conlleva una serie de decisiones individuales en el proceso de grupo sobre la aceptación o exclusión de alguien ( $y$ de hecho puede haber disidentes a los procesos de grupo que no acepten la decisión tomada por los demás).

3) El postulado sobre el conocimiento perfecto del estado de la estructura de la red por parte de los actores no es realista en absoluto. Mucho menos cuanto mayor sea la red estudiada. Es difícil en una red grande conocer el estado preciso de las relaciones entre personas. Tal vez sería más realista introducir el conocimiento de distancia 2, aunque incluso en este caso podemos dudar el conocimiento de los actores sobre las relaciones de sus vecinos, en el mejor de los casos es posible que éstos se hagan una "idea", no necesariamente cierta, del estado de la red cercana. También, dado el carácter voluntario, personal y más o menos privado de las relaciones de amistad, que puede independizarse del contexto inicial de interacción (Allan 1989), no siempre es posible conocer la existencia o la calidad de un lazo particular entre dos personas más allá de su mera relación cordial o 
amistosa incluso cuando su interacción es observable. Ciertos tipos de relaciones son más o menos visibles para los otros miembros de la red ${ }^{14}$.

Los postulados 4, 5 y 6 son más aceptables. 4) En el caso de relaciones de amistad se puede considerar que dentro del contexto en que los actores interactúan, éstos pueden escoger a sus amigos entre las relaciones posibles y disponibles. 5) Aunque los cambios no ocurran necesariamente uno a uno en la realidad sino también de forma simultánea, tampoco es un postulado teóricamente molesto. 6) El hecho de que ocurren cambios entre los momentos de observación de la red es perfectamente realista.

7) El postulado de que toda información relevante del pasado está contenida en el presente puede ser "corregido" mediante variables adicionales. Por ejemplo si tenemos motivos para pensar que la inestabilidad de una relación en el pasado (su alternancia entre presente y ausente) puede explicar los estados futuros de ésta (e.g. que las relaciones inestables tengan más probabilidad de ser eliminadas que las estables), se pueden crear variables que describan tal inestabilidad e incluirlas en el modelo.

8) El postulado de la intención no estratégica sino simplemente de satisfacción inmediata se adapta bien a las relaciones de amistad. En éstas, las estrategias estructurales a largo plazo, aunque puedan existir, están mal vistas. Sería ofensivo buscar la amistad de alguien, no por ésta misma, sino para establecer relaciones con otros o para otros fines. Así pues, en el caso de las relaciones de amistad, podríamos considerar que el modelo es válido para actores "de buena fe" o que hace como si todos los actores fueran de buena fe. Sin embargo, para otros tipos de relaciones los actores pueden tener estrategias relacionales de distancia 2 o más lejanas, a medio o a largo plazo. Tal vez sea posible modelar éstas a partir de variables seleccionadas por los investigadores.

A pesar de todas estas críticas, podemos considerar que éste modelo puede aportarnos conocimientos válidos sobre los procesos de emergencia de relaciones en una red. Tanto más si tenemos presentes sus limitaciones. Es

${ }^{14}$ iCuánto nos facilitaría la tarea a los analistas de redes sociales que esta suposición fuese cierta! 
posible "minimizar" los problemas que pueden dar tales supuestos mediante una selección cuidadosa de las variables que se incluyen en el modelo a partir de una reflexión teórica. Es más, estudiar de forma explícita los propios factores que van contra las suposiciones del modelo dentro de este marco formal general puede permitirnos aprender mucho sobre éstos.

Por otra parte, los autores del modelo no afirman que la supuesta evaluación u optimización de la posición que se entiende que realizan los actores sea necesariamente consciente, ni que se trate de un comportamiento intencionado. Lo único que afirman es que la red puede ser modelada como si cada actor tratase de obtener una configuración satisfactoria de sus relaciones. Esta manera de comprender la evolución de las redes sociales está en línea con el principio teórico sociológico del individualismo metodológico (ver Degenne y Forsé 1994) que Snijders Ilama aquí modelo aleatorio orientado por el actor (stochastic actor-oriented model). A partir de los resultados de la modelización se pueden encontrar las limitaciones o las preferencias de los actores cuando estas han sido tenidas en cuenta por el investigador e incluidas en el análisis.

Aparte de la función llamada de utilidad, SIENA también incluye una función de velocidad para tener en cuenta velocidades diferenciales en los cambios de relaciones (lo que permite estudiar hipótesis del tipo e.g. los hombres cambien sus amigos a mayor velocidad que las mujeres). También incluye una función de gratificación para dar cuenta de la importancia diferencial de ciertas variables a la hora de crear las relaciones o a la hora de eliminarlas, lo que permite estudiar efectos ligados a las inversiones relacionales (p. e. la reciprocidad puede tener un papel diferente respecto a la creación de relaciones que respecto a su destrucción).

El modelo debe ser construido con parsimonia estudiando sólo 2 o 3 efectos cada vez y eliminando los efectos no significativos. Los efectos estudiados se controlan mutuamente. Los resultados deben ser leídos como T-Test, esto es, para considerar que un efecto es significativo, el parámetro debe ser al menos dos veces mayor que la desviación típica ${ }^{15}$. Cuanto mayor es el

\footnotetext{
${ }^{15}$ Más precisamente si $t>1,65, p<0,10$, si $t>1,96, p<0,05$, si $t>2,53, p<0,01$ y si $t>3,29, p<0,00$
} 
parámetro respecto a la desviación típica, más seguros estamos de su significatividad. Sin embargo es difícil decir nada sobre la magnitud de los efectos o compararlos entre sí ya que ésta depende de la distribución de los valores en cada variable, y por lo tanto éstos son diferentes casi cada vez.

Así pues, gracias a SIENA vamos a poder estudiar la evolución de la red de los estudiantes Erasmus desde su situación inicial (T0), al poco de llegar a la universidad de Lille, hasta cuatro meses después (T1). Para ello necesitamos comenzar por describir el estado de la red de los estudiantes Erasmus en esos dos momentos.

\section{Descripción de la red Erasmus}

\subsection{Situación inicial TO, conocidos y amigos}

Dado que los estudiantes provenían en su mayoría de países y universidades diferentes, al poco de llegar, muchos de ellos no conocían apenas a nadie. La situación inicial presenta una matriz casi vacía. Sin embargo, algunos estudiantes conocían a los otros estudiantes provenientes de la misma universidad que ellos y, entre éstos, algunos ya eran amigos.

Simplemente mirando la Figura 3 realizada gracias a Pajek podemos observar, por ejemplo, que muchos de los estudiantes británicos ya se conocían antes de llegar a Lille (e.g. abajo en el centro, el grupo de nodos negros de Samuel, Ruth, las dos Sarah, Julie, Victoria, Tracey y Adrien eran todos estudiantes de matemáticas de la misma universidad. Más a la izquierda, los nodos negro Nathalie, Thu Hang, Katrina, Sonia, Claire, Johnatan y Warwick eran todos estudiantes de económicas). Unos cuantos italianos, algunos alemanes, algunos españoles, provenientes de las mismas universidades, también se conocían entre sí antes de llegar a Lille. Sin embargo sus relaciones no eran tan frecuentes como en el caso de los británicos.

\footnotetext{
Leyenda para todas las figuras:

Rojo: Españoles Azul oscuro: Griegos

Negro: Británicos Azul claro: Suecos

Naranja: Italianos Verde claro: Austriacos

Flechas negras: Relaciones dirigidas.

Líneas azules: Relaciones recíprocas.
}

Verde: Alemanes

Violeta: Belgas

Amarillo: Portugueses 
Figura 3. Conocidos en TO

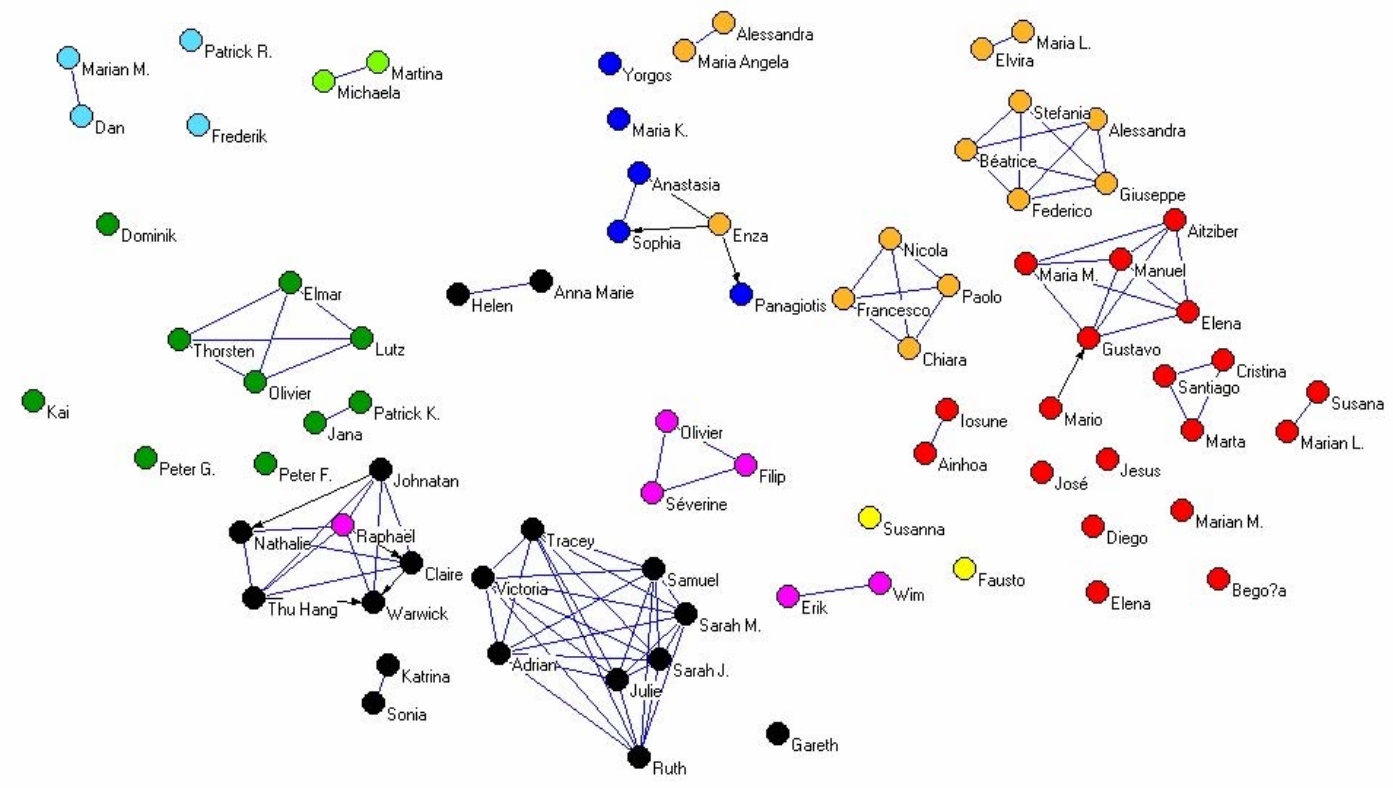

Figura 4. Amigos en To

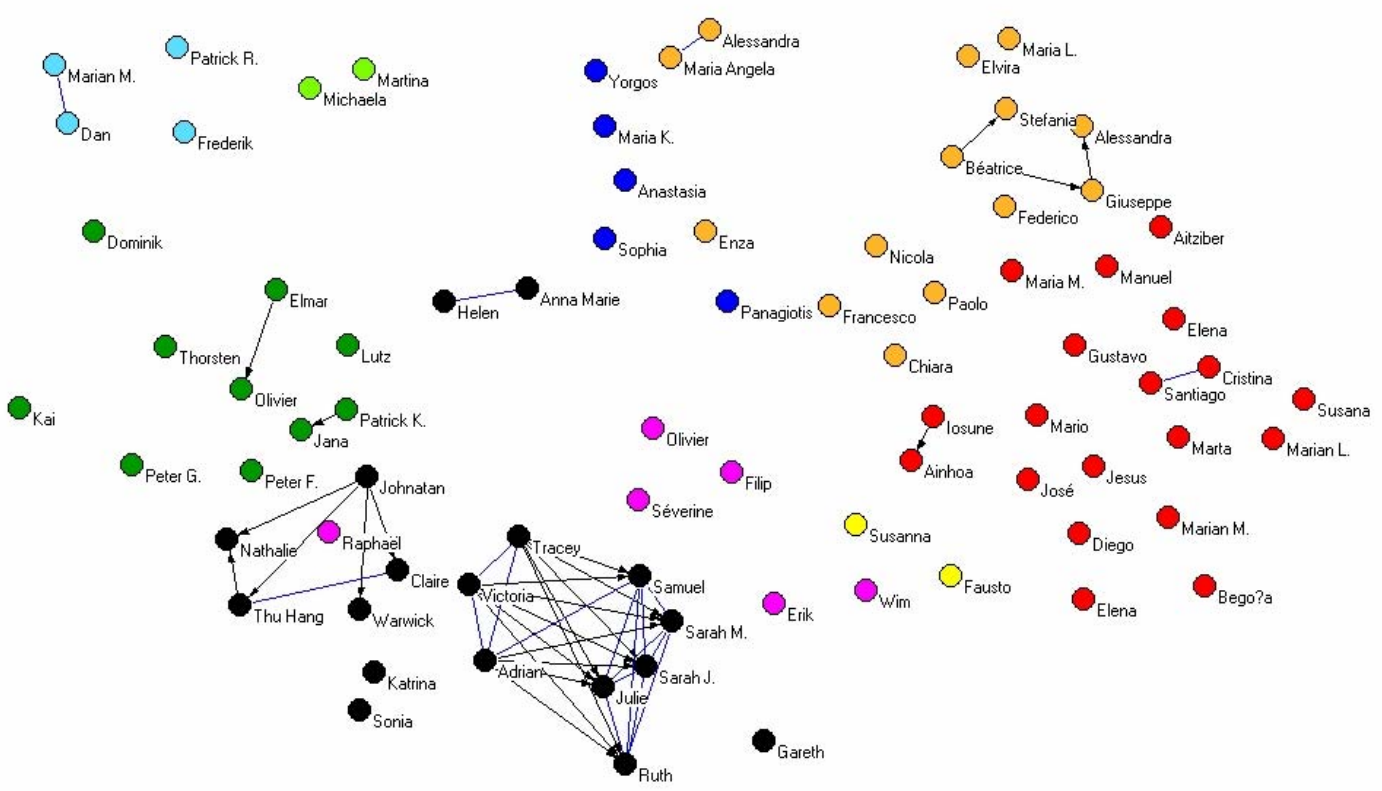


Si examinamos el grafo representando la matriz de amigos, vemos que está mucho más vacío. Se ven algunas díadas sueltas: Marian M. y Dan, Cristina y Santiago eran parejas, y Helen y Anne Marie (GB) amigas. Y algunas relaciones dirigidas de Patrick a Jana $(A)$, de Iosune a Ainhoa (E), de Elmar a Olivier (A), de Beatrice a Stefania y Giuseppe (I), y de éste último a Alessandra (I) eran amigos antes de llegar a Lille. Entre los 8 estudiantes de matemáticas británicos (Samuel, Ruth, las dos Sarah, Julie, Victoria, Tracey y Adrien) había bastantes relaciones de amistad preexistentes, de algún modo ya constituían un grupo antes de llegar. Entre los estudiantes británicos de económicas (Nathalie, Thu Hang, Katrina, Sonia, Claire, Johnatan y Warwick) también había algunas, aunque ese conjunto está mucho menos conectado.

Aunque las figuras de Pajek nos permiten tener una idea del aspecto general de la red, examinamos de manera sistemática algunas características generales de la red mediante Ucinet y Siena.

Tabla 3. Características de la red de amistad en To

\begin{tabular}{|l|c|c|}
\hline & Conocidos & Amigos \\
\hline Rango medio & 1.6 & 0.79 \\
\hline Rango de entrada & $0-7$ & $0-7$ \\
\hline Varianza del rango de entrada & 1.90 & 1.73 \\
\hline Rango de salida & $0-7$ & $0-7$ \\
\hline Varianza del rango de salida & 2.02 & 1.67 \\
\hline Densidad & 0.02 & 0.01 \\
\hline Reciprocidad & 0.988 & 0.992 \\
\hline Transitividad & 0.001 & 0.000 \\
\hline
\end{tabular}

Como se puede observar a partir de la Tabla 3, en T0 la red está casi vacía: el rango medio de conocidos por persona no alcanza a dos y el rango de amigos no llega a uno. El número de relaciones emitidas por los individuos varía de ninguna a siete (el grupo de matemáticos), tanto para los conocidos como para los amigos. Lo mismo sucede con las relaciones recibidas. La varianza de los rangos de entrada y de salida para relaciones recibidas y emitidas se encuentra en torno a dos. La densidad y la transitividad son muy bajas. Por otro lado la reciprocidad es muy alta, está claro que, en una situación en que tan poca gente se conoce y menos aún 
son amigos, los que lo son, suelen tener muy claro que la relación es mutua.

\subsection{Situación final T1, amigos y mejores amigos}

Tabla 4. Características de la red de amistad en T1

\begin{tabular}{|l|c|c|}
\hline & Amigos & Mejores amigos \\
\hline Rango medio & 11.68 & 3.0 \\
\hline Rango de entrada & $2-27$ & $0-8$ \\
\hline Varianza del rango de entrada & 5.56 & 1.21 \\
\hline Rango de salida & $0-35$ & $0-4$ (limitado a 4) \\
\hline Varianza del rango de salida & 7.61 & 1.82 \\
\hline Densidad & 0.15 & 0.04 \\
\hline Reciprocidad & 0.896 & 0.973 \\
\hline Transitividad & 0.012 & 0.001 \\
\hline
\end{tabular}

Como se podría esperar, la situación en T1, es muy diferente. En cuanto a los amigos se refiere, el rango medio ha aumentado hasta más de once amigos por persona, lo que se trata de una cifra bastante alta cuando se compara con otras redes de amistad de características similares (ver van de Bunt 1999 p. 119). Las relaciones emitidas varían de ninguna hasta 35, con una varianza de más de siete, y las relaciones recibidas varían desde dos hasta 27 con una varianza más baja, de más de cinco. Aunque la densidad sigue siendo relativamente baja (es raro que redes tan grandes sean muy densas) es siete veces mayor que la densidad inicial de conocidos. La transitividad también ha aumentado doce veces, y aunque es mucho más alta que al principio, resulta sorprendente su valor tan bajo (mucho más baja que en el ejemplo de van de Bunt 1999, p. 119). La reciprocidad sigue siendo muy alta, casi 0.9 , pero es levemente más baja que al principio, esto es normal, dada la sociabilidad mucho mayor y más difusa, es más fácil que haya algunos desacuerdos, olvidos o deslices al mencionar a los amigos de forma mutua. La Figura 5 ilustra bien el cambio que ha tenido lugar entre las dos redes. Ésta nos muestra en efecto una red mucho más densa y en la que intuyen agrupaciones, que más adelante veremos de forma sistemática. 
Figura 5. Amigos en T1

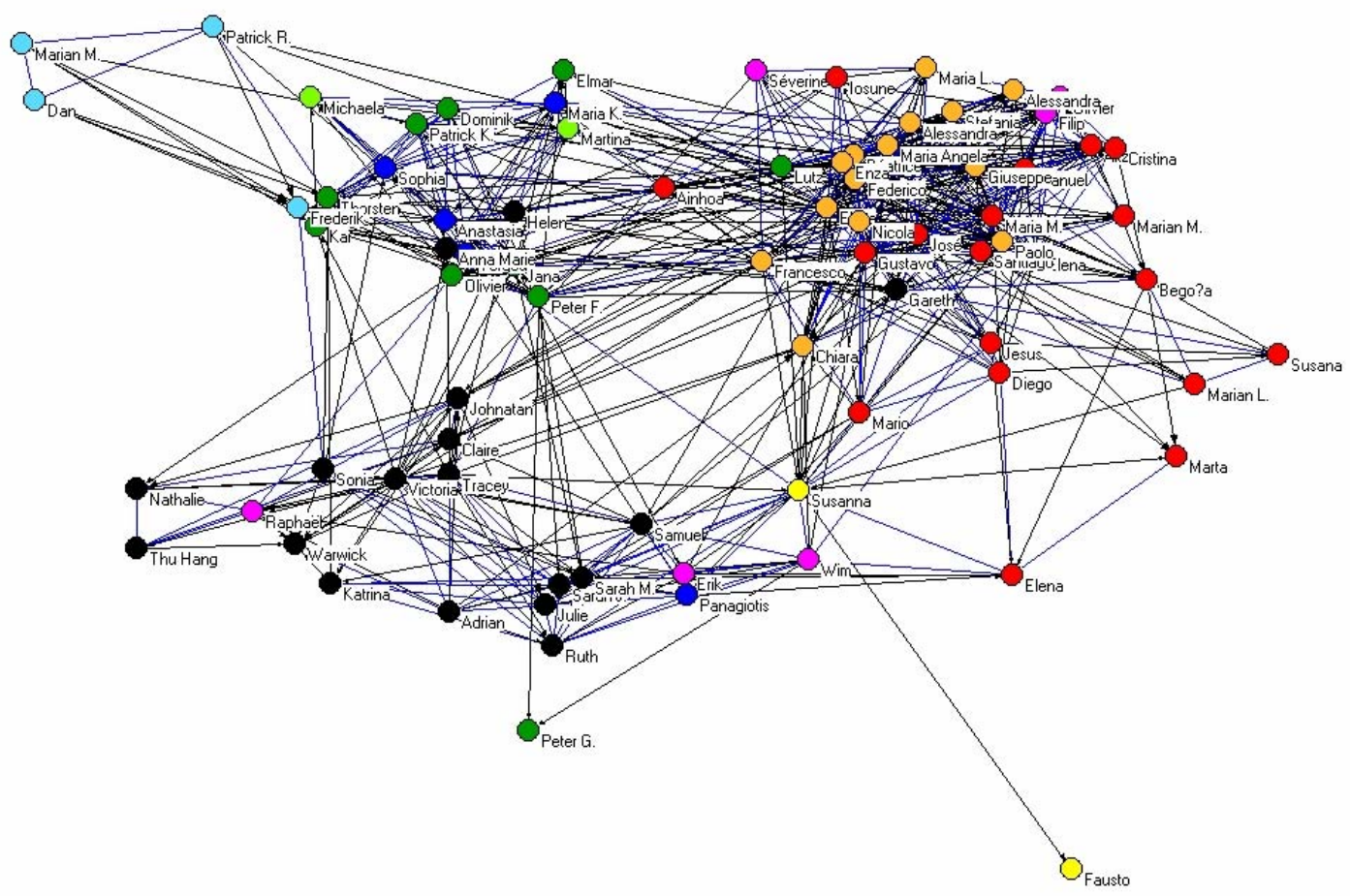

Figura 6. Mejores amigos en T1

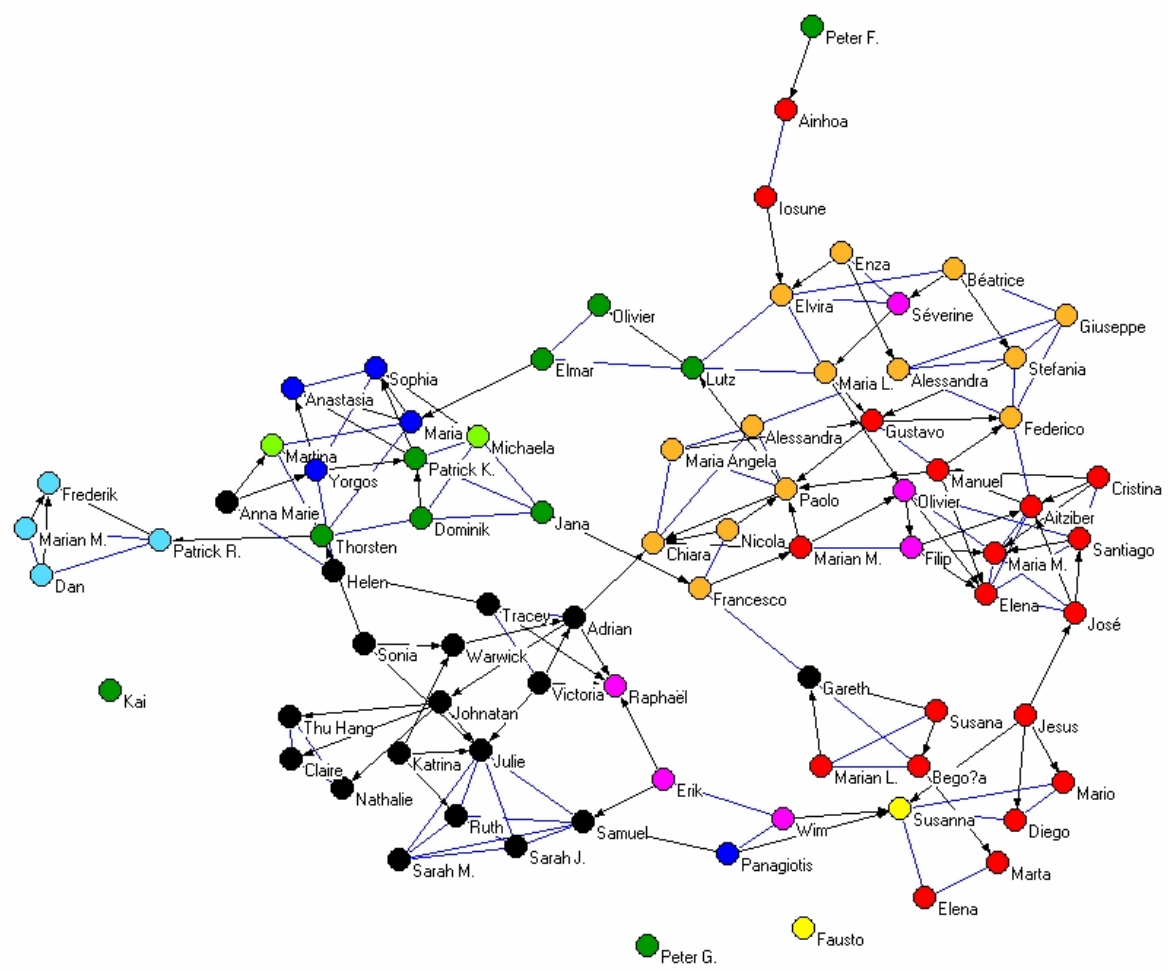


Si nos fijamos en los mejores amigos (Figura 6), vemos que se trata de una red mucho menos densa que la de amigos. El rango medio de mejores amigos es de 3. Los actores mencionaron de 0 a 4 mejores amigos (recordemos que el número de mejores amigos que se podía citar estaba limitado a 4) con una varianza de casi dos. A su vez, los actores fueron elegidos de 0 a 8 veces con una varianza de poco más de uno.

Dada la importancia acordada por la UE a la movilidad estudiantil en tanto que experiencia intercultural permitiendo desarrollar la solidaridad entre los pueblos, decidimos estudiar la estructura de las relaciones entre países subyacente a la red. Para ello calculamos la desviación entre la elección real de amigos de una nacionalidad por parte de ciudadanos de otra y la distribución esperada si la elección hubiera sido aleatoria, teniendo en cuenta la cantidad de ciudadanos de cada país presentes en la universidad.

Cálculo de la desviación:

$$
D=\frac{E R-E E}{E E} \times 100
$$

D representa la desviación. ER representa la elección real EE representa la elección esperada.

La Figura 7 nos muestra la estructura internacional de la red de amistad. Todas las nacionalidades mostraron una preferencia por ellas mismas, impresionante en el caso de los suecos, relativamente débil en el caso de los portugueses y belgas. Además, se puede observar en ella una distinción más o menos clara en tres bloques. El primero contiene a suecos, alemanes, austriacos y griegos. Si no fuera por la presencia de los griegos se podría calificar como "germánico". Se observa en este primer bloque una clique clara entre alemanes, austriacos y griegos. Ligados a los alemanes aparecen los suecos, que son correspondidos por parte de los por los alemanes pero en mucho menor medida (la reciprocidad está presente pero no es elevada). Los griegos tienen además una posición de intermediación con una relación recíproca débil hacia los portugueses del segundo bloque. 
Figura 7. Elección de amigos entre nacionalidades

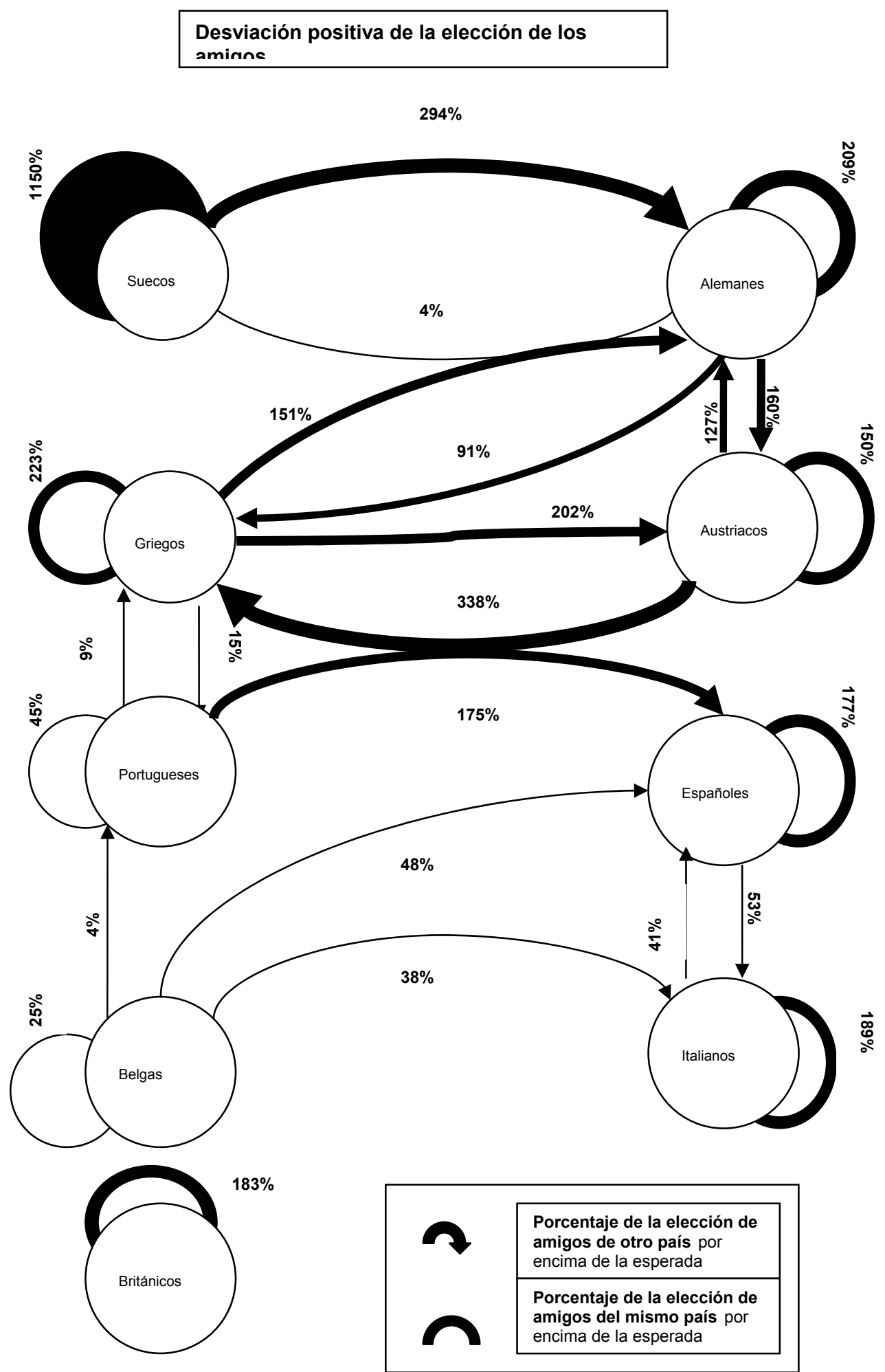


El segundo bloque contiene a portugueses, españoles, italianos y belgas. Se podría calificar a este segundo bloque de "latino" si no fuera porque los belgas eran de habla flamenca y no francesa. En este bloque se observa una cierta relación recíproca entre españoles e italianos, los portugueses con una clara preferencia por los españoles que no es recíproca, y los belgas con una leve preferencia no recíproca por españoles, italianos y portugueses. Los belgas son la nacionalidad cuyo modelo relacional se acerca más a una distribución aleatoria, por lo tanto una sociabilidad más "europea".

El tercer bloque estaba constituido por los británicos.

Este análisis sugiere una serie de pistas e interrogantes que incorporamos a nuestros análisis sobre la evolución de la red. Por ejemplo, la figura parece sugerir una cierta preferencia (con importantes excepciones) por países de rasgos culturales parecidos ies ello cierto o se trata de otros factores no visibles en este análisis? Si se da una preferencia por países de rasgos culturales parecidos, ¿cómo se puede explicar la presencia de griegos y belgas en tales bloques? Unos cuantos griegos (no todos) vivían cerca de algunos de los alemanes y austríacos ¿serán las oportunidades de interacción ligadas al lugar de residencia? Si es así, tal vez ello explique también el aislamiento de los británicos, cuya mayoría vivía alejada del campus universitario. Dado que tampoco todos los griegos vivían cerca de todos los alemanes y austríacos ¿habrá un efecto de "gregarización" a partir de un núcleo inicial?, esto es, ¿acaso una vez que los estudiantes identificaron grupos de nacionalidades que, por los motivos que fuera, se relacionaban entre sí, se acomodaron a estos grupos? ¿Y los belgas? ¿Puede ser su mejor dominio de más idiomas lo que explique tanto su sociabilidad "europea" como su posición en el segundo bloque? Los análisis que llevamos a cabo con Siena permitieron desenmarañar los diferentes efectos que intervinieron en la formación de la red de amistad. 
Tabla 5. Factores estudiados

\begin{tabular}{|l|l|}
\hline Factor & Variable \\
\hline Oportunidades de contacto & Lugar de residencia \\
\hline & Mismos estudios \\
\hline & Uso del e-mail \\
\hline Similitud Individual & Asociación de integración Erasmus \\
\hline & Mismo sexo \\
\hline Similitud Internacional & Misma nacionalidad \\
\hline & Familia lingüística \\
\hline & Tradición religiosa \\
\hline Cultura & Norte/Sur \\
\hline & Actividad por nacionalidad \\
\hline Red & Popularidad por nacionalidad \\
\hline & Reciprocidad \\
\hline & Cierre de la red \\
\hline & Tener pareja \\
\hline & Alternativas de amigos no erasmus \\
\hline & Amigos locales conocidos de antes \\
\hline Inversión & No de estudiantes de la misma universidad \\
\hline & Duración de la estancia \\
\hline & Misma universidad \\
\hline & Conocimiento de los idiomas \\
\hline Convergencia a los grupos & Conocimiento de los idiomas minoritarios \\
\hline Conformidad o exotismo & Nacionalidad X reciprocidad \\
\hline & 3 zonas entre naciones X reciprocidad \\
\hline &
\end{tabular}

\section{La emergencia de una red de amistad}

En esta sección examinamos los factores intervinieron en el desarrollo de la red de amistad a partir de la situación inicial (ver figuras 1 y 2) hasta la situación final (ver figuras 3 y 4). Dado que la matriz inicial de los amigos está demasiado vacía para llevar a cabo el estudio ${ }^{16}$, tomamos como punto de partida la red de conocidos. Desde un punto de vista temporal, entonces estudiamos dos transiciones: 1) la transición a partir de la red de conocidos hasta la red de amigos y 2) la transición a partir de la red de conocidos hasta la red de mejores amigos. Examinamos las dos transiciones por separado. En ambas transiciones examinamos la incidencia de los factores enunciados en la tabla 5.

${ }^{16}$ Se trata de una cuestión puramente técnica, los análisis de SIENA no convergían a partir de la matriz de amigos. Por otro lado, desde un punto de vista teórico se puede aceptar perfectamente que una red 


\subsection{Transición de conocidos a amigos}

Para dar cuenta de la "cantidad de cambio" que ha tenido lugar entre la red de conocidos en T0 y la red de amigos en T1 utilizamos el índice de Jaccard (Jaccard 1900, van de Bunt 1999). Según éste la cantidad de cambio se expresa así: J = 1 - n11 / (n11+n01+n10). El valor de J queda comprendido entre 0 y 1.

La tabla que sigue muestra lo que sucedió con las relaciones entre T0 y T1. De todas las relaciones posibles entre los 80 actores, 5383 no existían en T0 y tampoco existían en T1. De T0 a T1 810 relaciones de amistad fueron creadas, 3 relaciones fueron retiradas y 124 fueron mantenidas.

\begin{tabular}{|c|c|c|c|c|}
\hline Transición & $0->0$ & $0->1$ & $1->0$ & $1->1$ \\
\hline $\begin{array}{c}\text { Número de } \\
\text { lazos }\end{array}$ & 5383 & 810 & 3 & 124 \\
\hline
\end{tabular}

El índice de Jaccard J= 1-124 / $(124+810+3)=0.87$ muestra que ha tenido lugar un cambio enorme entre T0 y T1. Mucho más interesante que "cuanto" cambio ha tenido lugar es examinar los factores que tuvieron un rol en el establecimiento de la red de amigos.

Tabla 6. Factores de elección de los amigos

\begin{tabular}{|c|c|c|c|c|c|}
\hline Efecto & Tipo & Parámetro & $\begin{array}{c}\text { Desviación } \\
\text { típica }\end{array}$ & Relación & Factor explicado \\
\hline $\begin{array}{l}\text { Razón de } \\
\text { cambio }\end{array}$ & Interno & 11,18 & 0,24 & 47 & \\
\hline Densidad & Interno & $-1,40$ & 0,54 & -3 & \\
\hline $\begin{array}{l}\text { Lugar de } \\
\text { residencia }\end{array}$ & $\begin{array}{c}\text { Oportunid } \\
\text { ad }\end{array}$ & 0,71 & 0,10 & 7 & \\
\hline Nacionalidad & $\begin{array}{l}\text { Similitud } \\
\text { individual }\end{array}$ & 0,30 & 0,11 & 3 & $\begin{array}{c}\text { Distancias 2, } \\
\text { Idiomas, Idiomas } \\
\text { minoritarios }\end{array}$ \\
\hline $\begin{array}{c}\text { Actividad de } \\
\text { los } \\
\text { británicos } \\
\end{array}$ & Cultura & $-20,61$ & 3,75 & -5 & $\begin{array}{l}\text { Similitudes } \\
\text { nacionales }\end{array}$ \\
\hline Reciprocidad & Red & 2,54 & 0,36 & 7 & \\
\hline Transitividad & Red & 0,75 & 0,17 & 4 & Mismos estudios \\
\hline 3 zonas & $\begin{array}{c}\text { Agrupació } \\
\mathrm{n}\end{array}$ & 0,52 & 0,19 & 3 & Similitud religiosa \\
\hline $\begin{array}{l}3 \text { zonas } x \\
\text { reciprocidad }\end{array}$ & $\begin{array}{l}\text { Valor de } \\
\text { lo exótico }\end{array}$ & $-1,31$ & 0,28 & -5 & $\begin{array}{l}\text { Nacionalidad X } \\
\text { reciprocidad }\end{array}$ \\
\hline
\end{tabular}


La razón de cambio y la densidad son efectos, por decirlo de alguna manera, inherentes al modelo. Hay que incluirlos siempre cuando se utiliza SIENA y son más que nada efectos de control de los que no se puede decir gran cosa. El cambio entre las dos descripciones de la red es significativo. La densidad, como suele ser frecuente, es negativa, lo que quiere decir que la red no tiende a hacerse muy densa.

Los factores sustantivos que se mostraron significativos para el establecimiento de la amistad en esta red fueron los siguientes.

\subsubsection{Oportunidades}

La única variable ligada a la estructura de oportunidades de interacción significativa es el lugar de residencia de los estudiantes ( 7$)^{17}$. Muchas de las relaciones de amistad se desarrollaron probablemente ligadas a los ritmos de la vida cotidiana. Los estudiantes Erasmus, por otra parte alejados de sus familias, desarrollaron "núcleos de vida" con otros estudiantes Erasmus que vivían cerca, y se organizaban para comer y cenar juntos y compartir las tareas ligadas a ello ${ }^{18}$. Esta auto selección para la vida cotidiana ocurría a pesar de que los estudiantes Erasmus eran minoritarios en todas las residencias en la universidad.

Los estudios, sin embargo, lo cual fue una sorpresa, no se mostraron estructuradores de la interacción entre los estudiantes Erasmus. No parecen tener importancia ni como contexto de interacción ni como similitud preferible. Tampoco fueron estructuradoras de las relaciones de amistad la pertenencia a una asociación de integración para los estudiantes Erasmus y la utilización de la sala para el correo electrónico. Si lo fueron fue tal vez sólo para el primer encuentro, pero no para la selección de los amigos específicos.

\footnotetext{
17 La cifra entre paréntesis hace referencia a la relación entre el valor del parámetro y su desviación típica en este caso, el valor del parámetro es siete veces mayor que el de su desviación típica.

18 Esta experiencia era a menudo valorizada en los comentarios de los estudiantes. Una estudiante griega, por ejemplo, decía "he compartido el desayuno con ' $x$ ' e ' $y$ ' en pijama $y$ sin peinar, hemos comido chocolate juntos para llorar nuestras penas de amores, nos hemos peleado por fregar los platos... ¿cómo voy a pensar en ellos como extranjeros?, ison como mis hermanos!".
} 


\subsubsection{Similitud individual e internacional}

Estudiamos dos variables de similitud individual y tres de similitud entre países. En cuanto a las variables de similitud individual, tuvimos una gran sorpresa con la incidencia del género en la selección de amigos. Las investigaciones que estudian la amistad y el género suelen dar cuenta de la preferencia por tener amigos del mismo sexo (Derlaga y Winstead 1986, Bidart 1993, Requena 1994), incluidos los estudios relativos a estudiantes universitarios de edades similares a los estudiados aquí (Estudiantes holandeses y japoneses, ver van de Bunt 1999, Zeggelink et al 1997, 1999, Hiramatsu 1999, van Duijn et al 2002). La hipótesis que adelantamos aquí para explicar el resultado diferente en este caso es una hipótesis de control lateral por parte de la red. En el propio país e incluso cuando un estudiante se traslada (el caso de los holandeses, ver van de Bunt 1999, Zeggelink et al 1997, 1999, van Duijn et al 2002) es más probable que de modo consciente 0 inconsciente el estudiante se sienta "vigilado" sobre sus elecciones de amistades por padres, madres, amigos, novio o novia. En el caso de los estudiantes Erasmus, el control normativo no es efectivo, ya que tales relaciones "controladoras" están alejadas del contexto actual, tanto de modo geográfico como cultural. Los estudiantes Erasmus se encuentran en un contexto en que se ven rodeados por otros individuos "no controlados" y que probablemente tampoco actúan de forma "controladora"19. Así pues, parece que de algún modo la experiencia Erasmus tiene un aspecto liberador del control social impuesto por las redes personales habituales que exigen conformarse a normas y roles, y que sancionan cuando esto no ocurre $^{20}$.

\footnotetext{
${ }^{19}$ Como dato curioso, aquellos estudiantes que tenían novia o novio en su país de origen eran más propensos a tener también novia o novio en Lille... Aunque aquí describimos "inocentes" relaciones de amistad el mecanismo de control puede ser el mismo. Para una ilustración divertida de este fenómeno y, en general de la sociabilidad Erasmus, es muy aconsejable ver la película L'auberge espagnol de Cédric Klapisch (Francia 2002).

20 Otros análisis, que solo mencionamos aquí a modo de ilustración, apoyan esta idea de experiencia liberadora: durante el periodo Erasmus las redes personales de los hombres y mujeres de esta muestra son mas similares, por ejemplo en tamaño y actividad, que en el país de origen, en que las mujeres tienen redes personales mas pequeñas y con menores frecuencias de interacción que las de los hombres.
} 
La preferencia por amigos de igual nacionalidad se mostró, tal como esperábamos, significativa (3). Además, la preferencia por la misma nacionalidad absorbió el efecto del idioma. Es decir que el hecho de hablar mejor o peor el idioma de los alteri (o al revés) incluso cuando se trata de lenguas minoritarias (como el griego), no parece tener incidencia en la selección de los amigos.

Estudiamos también si existían rasgos específicos por países de preferencia por la propia nacionalidad por encima de la media, de mayor sociabilidad o de mayor popularidad. Encontramos un único efecto y muy significativo: la falta de actividad de los estudiantes británicos. A la vista del resultado se diría que, aunque crearan relaciones de amistad entre los dos momentos observados, crearon muchas menos que los demás estudiantes e incluso debieron romper alguna. Los británicos se muestran los elementos menos sociables de la red, no sólo respecto a las demás nacionalidades (como ya lo mostraba la figura 7) sino en general e incluso controlando tanto por el lugar de residencia como por el número de amigos inicial.

A partir de las nacionalidades de los individuos, creamos variables de similitud entre países a partir de tres criterios para ver si el hecho de que las personas pertenecieran a países con rasgos culturales más o menos parecidos aumentaba la probabilidad de selección de amigos de esos países. La similitud por familias lingüísticas (germánicas, latinas, griego) no se mostró significativa. Tampoco se mostró significativa la distinción geográfica norte/sur entre los países. La similitud según la tradición religiosa (protestantes, católicos, ortodoxos) de los países sí se mostró algo significativa, pero este efecto fue absorbido por la tendencia a la agrupación según un núcleo inicial de países (3).

De alguna manera el hecho de que estos resultados no sean significativos es muy esperanzador, ya que éstos implican que para establecer relaciones personales de confianza mutua, solidaridad, entendimiento, amistad..., en un contexto propicio, las diferencias culturales o las dificultades idiomáticas no son una barrera. Una vez que es posible establecer relaciones de amistad entre personas de países incluso de rasgos diferentes, se abre la puerta a 
efectos todavía más profundos de la amistad, como procesos de reducción de los estereotipos y prejuicios, de descategorización (Pettigrew y Meertens 1995, Brewer y Gaertner 2001, de Federico 2002b) y tal vez a cambios en los sistemas de identificación (de Federico 2001, 2002b).

Encontramos incluso una bonita preferencia por las relaciones inhabituales o exóticas, puesto que, aunque los actores prefieran tener en general relaciones con personas de su misma nacionalidad o dentro del grupo de nacionalidades que hacia el que se converge, cuando se dan relaciones transnacionales o trans-grupo, éstas son significativamente más recíprocas que las relaciones dentro de la misma nacionalidad o grupo (-5). Este indicador también es alentador respecto la fuerza del lazo y por lo tanto al futuro de la relación, ya que las relaciones recíprocas denotan una mayor intimidad y probabilidad de duración de la relación. Este resultado incluso relativiza la importancia de la similitud nacional (es incluso más significativo que éste): parece que es más fácil o más natural relacionarse con personas de igual nacionalidad, sin embargo una vez que se establecen relaciones entre nacionalidades y entre grupos diferentes, éstas son preferidas y más fuertes en términos diádicos ${ }^{21}$.

\subsubsection{Red}

Las relaciones preexistentes pueden orientar las relaciones futuras funcionando como limitadoras de futuras relaciones (como ya hemos visto que no es el caso respecto a las relaciones entre géneros en este contexto), como oportunidades para acceder a nuevas relaciones (los amigos de mis amigos se convierten en mis amigos), también como alternativas (si ya tengo un amigo de tales características, tal vez no necesite tener otro). Estudiamos varias variables para examinar estos aspectos de interdependencia entre las relaciones.

La reciprocidad es una interdependencia puramente diádica, indica una preferencia por la mutualidad en la relación de amistad. En efecto, asistimos

\footnotetext{
${ }^{21}$ Aunque se trata de un tipo de relación diferente, encontramos también que las relaciones amorosas tendían a ser más frecuentemente entre personas de nacionalidades diferentes que entre personas del mismo país.
} 
a la gran significatividad de la mutualidad (7), esperable en las relaciones personales afectivas como son las relaciones de amistad, en el desarrollo de las relaciones en la red. La transitividad, o la tendencia de los amigos de mis amigos a devenir mis amigos también es significativa (4).

Encontramos por otra parte que la hipótesis de las alternativas no parece funcionar. El hecho de tener más amigos fuera de la red Erasmus no parecía inhibir la sociabilidad con estudiantes Erasmus. Los individuos parecen crear relaciones de amistad sin "contar" cuántas tienen ya y de qué tipo. Sus preferencias respecto al número de amigos ideal o sus habilidades para crear amistades pueden ser muy variables. Por otra parte, los individuos que tenían pareja (ya fuera en Lille o en su país de origen) no parecen ni más ni menos sociables que el resto. El hecho de tener amigos en Lille antes de llegar tampoco parece incidir en la mayor o menor sociabilidad respecto al grupo de estudiantes Erasmus y el tamaño del grupo de estudiantes llegados de la misma universidad tampoco afecta la sociabilidad.

\subsubsection{Inversión}

Los efectos de costes e inversión que hubiéramos podido imaginar, tampoco son significativos. La duración de la estancia no afecta la sociabilidad de los estudiantes, éstos no se involucran más con sus amigos si se van a quedar más tiempo, ni tampoco buscan amigos que se vayan a quedar tanto tiempo como ellos. Tampoco buscan relacionarse con personas de la misma universidad que ellos con quienes podrían más fácilmente continuar la relación después del periodo en el extranjero.

El mejor dominio de ciertos idiomas, lo que facilitaría la comunicación y reduciría los "costes", el esfuerzo, para comunicarse de forma directa y profunda, tampoco tienen ninguna importancia. Con una base suficiente para comprenderse, los estudiantes parecen escoger sus amigos según otros criterios y están dispuestos a hacer el esfuerzo de comunicarse con ellos, y no al revés.

Así pues parece (lo cual es un alivio, más tratándose de relaciones afectivas, de solidaridad y confianza) que los actores no son tan racionales y 
calculadores a la hora de establecer sus relaciones, mirando los costes y beneficios y los retornos futuros de las inversiones en las relaciones, como algunos autores (o sus aproximaciones) pretenden (van de Bunt 1999). De Vos (2002) hace esta misma crítica a Williamson (1993).

\subsection{De conocidos a mejores amigos}

Pero qué sucede con una relación de mayor intensidad e intimidad, con los mejores amigos. ¿Cuáles son los factores que inciden en la elección de los mejores amigos? ¿Se ven las relaciones más profundas ligadas a los mismos procesos y mecanismos? Se podría esperar que, dado que se trata de relaciones más intensas, personales y privadas, la mutualidad tenga una importancia todavía mayor que para los amigos. También que la elección esté menos ligada a la estructura de oportunidades de interacción: aunque sea necesario encontrarse al menos una vez para conocerse, y la interacción frecuente facilitada permita conocerse mejor, probablemente las personas sean más selectivas con los mejores amigos y estén dispuestas a hacer más esfuerzos para verse y mantener la relación. Por lo demás, dejémonos sorprender y examinemos a continuación.

\begin{tabular}{|l|l|l|l|l|}
\hline Transición & $0->0$ & $0->1$ & $1->0$ & $1->1$ \\
\hline Número & 6030 & 163 & 50 & 77 \\
\hline
\end{tabular}

De entrada, a partir del índice de Jaccard $\mathrm{J}=0.74$, vemos que también ha tenido lugar un gran cambio en la transición de conocidos a mejores amigos, aunque menor que el que tuvo lugar de conocidos a amigos. Si se mira en detalle, se puede observar que hay muchas menos relaciones nuevas (163) que respecto a los amigos (810) y también, lo cual es esperable, hay muchas menos relaciones que, a partir de un mero conocimiento, llegaran a desarrollarse hasta mejores amigos (50, mientras que sólo 3 conocidos no se convirtieron en amigos).

Vemos que, salvo una sustitución, los mismos factores que tuvieron importancia para la emergencia de las relaciones de amistad son también significativos para elegir a los mejores amigos, aunque hay variaciones interesantes en su significatividad. A estos se añaden además tres factores nuevos. 
Tabla 7. Factores de elección de mejores amigos

\begin{tabular}{|c|c|c|c|c|c|}
\hline Efecto & Tipo & Parámetro & $\begin{array}{c}\text { Desviación } \\
\text { típica }\end{array}$ & Relación & $\begin{array}{c}\text { Factor } \\
\text { explicado }\end{array}$ \\
\hline Razón de cambio & Interno & 11,51 & 1,98 & 6 & \\
\hline Densidad & Interno & $-2,39$ & 0,22 & -11 & \\
\hline $\begin{array}{l}\text { Lugar de } \\
\text { residencia }\end{array}$ & $\begin{array}{c}\text { Oportunida } \\
\mathrm{d}\end{array}$ & 0,51 & 0,13 & 4 & \\
\hline Nacionalidad & $\begin{array}{l}\text { Similitud } \\
\text { individual }\end{array}$ & 0,62 & 0,18 & 3 & $\begin{array}{c}\text { Idiomas, } \\
\text { Idiomas } \\
\text { minoritarios }\end{array}$ \\
\hline $\begin{array}{l}\text { Popularidad de } \\
\text { los británicos }\end{array}$ & Cultura & $-1,11$ & 0,19 & -6 & \\
\hline Popularidad sur & Cultura & 0,46 & 0,21 & 2 & \\
\hline Reciprocidad & Red & 3,21 & 0,28 & 11 & \\
\hline Distancias 2 & Red & $-1,17$ & 0,13 & -9 & \\
\hline $\begin{array}{l}\text { Popularidad } \mathrm{x} \\
\text { tener pareja }\end{array}$ & Red & $-0,37$ & 0,11 & -3 & $\begin{array}{c}\text { Pareja en el } \\
\text { país de } \\
\text { origen }\end{array}$ \\
\hline $\begin{array}{c}\text { Actividad } x \mathrm{n}^{\circ} \text { de } \\
\text { estudiantes de la } \\
\text { misma } \\
\text { universidad }\end{array}$ & Red & $-0,13$ & 0,06 & -2 & \\
\hline $\begin{array}{c}\text { Actividad x } \\
\text { duración de la } \\
\text { estancia }\end{array}$ & Inversión & $-0,34$ & 0,16 & -2 & \\
\hline 3 zonas & Agrupación & 0,41 & 0,12 & 3 & $\begin{array}{l}\text { Similitud } \\
\text { religiosa } \\
\end{array}$ \\
\hline $\begin{array}{l}3 \text { zonas } x \\
\text { reciprocidad }\end{array}$ & $\begin{array}{l}\text { Valor de lo } \\
\text { exótico }\end{array}$ & $-0,57$ & 0,18 & -3 & $\begin{array}{c}\text { Nacionalidad } \\
\text { X } \\
\text { reciprocidad }\end{array}$ \\
\hline
\end{tabular}

\subsubsection{Oportunidades}

De nuevo encontramos que el lugar de residencia es significativo para la elección de los mejores amigos, aunque su nivel de significatividad se ha reducido (de 7 a 4). Se diría que la elección de los mejores amigos está algo menos sujeta a limitaciones circunstanciales, lo cual coincide con nuestras expectativas.

\subsubsection{Similitud}

La similitud por nacionalidades es significativa en igual medida (3). La similitud religiosa se mostró de nuevo significativa, pero absorbida una vez más por la tendencia a la agrupación según un núcleo inicial de países. Esta tendencia a la agrupación es igualmente significativa (3). 
La preferencia por relaciones inhabituales 0 exóticas sigue siendo significativa, aunque su significatividad se ha reducido (de 5 a 3 ).

Encontramos dos efectos ligados a aspectos culturales sobre la sociabilidad. Los británicos, que respecto a las relaciones de amistad se mostraban poco activos, en este caso se muestran poco populares, es decir que son muy significativamente no escogidos como mejores amigos (-6). Por el contrario los europeos del sur son escogidos de forma significativa (2) más a menudo como mejores amigos. Tal vez los rasgos culturales relativos a la sociabilidad hacen que estos sean más acogedores, más simpáticos, más sociables y por lo tanto preferidos en las relaciones más profundas.

\subsubsection{Red}

La reciprocidad se ha hecho mas significativa todavía (de 7 a 11), tal como esperábamos, y el efecto de cierre de la red (en este caso la no preferencia de distancias 2) también ha aumentado su significatividad (de 4 a 9). Tal vez, dado que los mejores amigos son una selección más fina de las personalidades más afines, es más probable que a su vez, estos sean más parecidos y afines entre ellos, o que se pase particularmente más tiempo con ellos, con lo cual éstos acaben conociéndose también muy bien entre sí.

Encontramos dos efectos adicionales relativos a las relaciones preexistentes como orientadoras de las relaciones futuras. Por una parte, los individuos con pareja (ya sea en Lille o en su país de origen) son menos "populares", menos elegidos como mejores amigos. Tal vez los demás consideraban que por sus otras obligaciones o preferencias afectivas estaban ocupados y por lo tanto eran percibidos como menos disponibles. Los emparejados, por su parte, no se mostraron menos activos que los demás.

\subsubsection{Inversión}

Encontramos un efecto opuesto a las ideas de la inversión. Contrariamente a lo que se hubiera podido esperar, según la idea de que aquellos individuos con un futuro relacional más largo invertirían más en las relaciones, los estudiantes que se quedaron menos tiempo eran más activos, escogían más mejores amigos. Tal vez la "inversión" debiera ser considerada al revés: 
dado lo estupendo de tener amigos, al ser tres meses un periodo breve, hay que aprovechar para establecer más y mejores contactos.

\section{Conclusiones, sugerencias}

En este artículo hemos estudiado las relaciones de amistad creadas por estudiantes Europeos participando en un programa de intercambio Erasmus.

Examinamos hipótesis sobre la amistad referentes a las oportunidades de contacto, la similitud, los roles y los efectos de las redes como limitadoras y/o posibilitadoras de nuevos contactos, así como controladoras de las normas que se aplican.

La primera constatación ha sido que el factor más estructurador de la formación de relaciones de amistad es el rol sociológico de extranjeros que ocupan los estudiantes Erasmus de manera temporal. La similitud respecto a este rol da cuenta de la elección del $80 \%$ de todos los amigos. Además, la ocupación de este rol determina qué similitudes son relevantes y cuales no, y por la distancia que este rol implica respecto a las relaciones preexistentes, qué tipo de relaciones, en este caso, no son controladas.

A partir de ésta, podemos argüir que los estudios de la psicología social en que, para los casos de estudiantes de intercambio, se invalida la hipótesis de que el contacto permite mejorar los sentimientos y estereotipos respecto a otros países, no son aceptables. Estos estudios tienden a pasar por alto el hecho de que los estudiantes de intercambio, por su propia posición sociológica de extranjeros, tienen un muy escaso contacto con los estudiantes locales incluso cuando las condiciones son supuestamente favorables para ello. Sin embargo las relaciones de amistad transnacionales son frecuentes y se pueden estudiar sus efectos allí donde éstas tienen lugar, es decir, entre estudiantes de intercambio provenientes de países diferentes. De hecho, uno de los resultados de Stroeber et al (1988) es que la experiencia de un intercambio, aunque no mejore, e incluso pueda empeorar la imagen del país de acogida, induce a reducir las generalizaciones sobre los estereotipos que se aplican tanto al país de acogida como a muchos otros países, lo que podría ser un efecto del 
contacto con personas de esos países. Este tipo de efectos merecen una atención más detenida por parte de los futuros estudios sobre estudiantes participando en intercambios.

Dada la enorme auto selección de los estudiantes de intercambio, reconstruimos la red de relaciones de amistad entre éstos, lo cual nos permitió a su vez estudiar los efectos estructurales de forma sistemática. Utilizamos el modelo de análisis longitudinal para redes totales SIENA, dado que se adapta bien a las relaciones de tipo voluntario como las de amistad. Nos hemos distanciado respecto a los postulados del modelo que suponen un actor evaluador que escoge sus relaciones de forma independiente a los demás y a partir de una información perfecta de la red. Admitimos que éstos sean necesarios para los procedimientos heurísticos, pero tanto los conocimiento de que disponemos en la literatura sociológica y antropológica sobre la amistad, como las propias conclusiones del presente estudio, contradicen gran parte de los postulados. Sin embargo el procedimiento representa una herramienta válida para estudiar los procesos dinámicos de redes totales de carácter más o menos voluntario.

Gracias al empleo de este modelo, identificamos los factores más importantes que estructuraron la emergencia de relaciones de amistad entre los estudiantes Erasmus, lo cual tiene su interés y consecuencias para los departamentos de Relaciones Internacionales que quisieran favorecer una mejor integración de los estudiantes, tanto respecto a los estudiantes locales, como de forma cosmopolita respecto a los demás estudiantes de intercambio. El contexto de sociabilidad más importante y estructurador parece ser el lugar de residencia, probablemente dada la interdependencia de los estudiantes para llevar a cabo las diferentes tareas cotidianas de manera más agradable, para lo cual éstos se constituían de manera espontánea en "núcleos de convivencia" dentro de las residencias. Los estudios sin embargo no se mostraron un contexto favorable de sociabilidad. Esto es probablemente debido a la dispersión a que se ven forzados en muchas ocasiones los estudiantes Erasmus, que deben tomar sus clases en cursos diferentes, lo que limita sus posibilidades de interactuar con las mismas personas de forma cotidiana y repetida, de compartir los 
mismos obstáculos y de crear interdependencias múltiples en los trabajos colectivos. La similitud por nacionalidad se mostró un factor de preferencia para la sociabilidad difusa, sin embargo las similitudes culturales (lingüística, religiosa, norte vs. sur) entre nacionalidades no se mostraron significativas e incluso encontramos una bonita preferencia por relaciones exóticas (con personas de distinta nacionalidad y con personas de grupos de nacionalidades diferentes) para las relaciones recíprocas. Encontramos también efectos significativos de reciprocidad (mutualidad en la elección de los amigos) y de transitividad (los amigos de mis amigos son mis amigos). Por otra parte, los europeos del sur (Italianos, Españoles, Portugueses y Griegos) se mostraron preferidos como mejores amigos y los Británicos se mostraron menos sociables y menos escogidos como mejores amigos.

Combinando estos hallazgos se podría sugerir a los departamentos de Relaciones Internacionales que tengan el interés y la capacidad de organizar el alojamiento de los estudiantes Erasmus: 1) Que intenten situar a estudiantes Erasmus en situaciones de interdependencia con estudiantes locales, en los pisos de estudiantes o en los núcleos de convivencia de las residencias. 2) Que intenten situar en los mismos lugares de residencia a personas de nacionalidades diferentes. Permitir que haya 2 personas del mismo país está bien para que puedan apoyarse, pero, cuando ello fuera posible, sería conveniente que las personas cohabitando un mismo lugar de residencia sean lo más diversas posible. Dado que las relaciones se crean en los lugares de residencia y que las relaciones trans-nacionales son preferidas y tienen más posibilidades de durar una vez establecidas, este sería un contexto propicio para producir relaciones trans-nacionales duraderas. 3) En particular sería interesante dispersar lo más posible, por motivos opuestos, a los europeos del sur y a los británicos. A los europeos del sur porque, dada su mayor sociabilidad, pueden convertirse en puentes relacionales entre países $y$, al hacer de sus amigos los amigos de sus amigos, contribuir a un mayor cosmopolitismo de la red. A los británicos para tratar de romper su aislamiento. 


\section{Anexo}

Tabla 8. Distribución de las nacionalidades

\begin{tabular}{|c|c|c|c|c|}
\hline Nacionalidad & No Población & \% Población & No Muestra & \% Muestra \\
\hline Españoles & 19 & $18 \%$ & 19 & $24 \%$ \\
\hline Británicos & 24 & $23 \%$ & 18 & $23 \%$ \\
\hline Italianos & 17 & $17 \%$ & 14 & $18 \%$ \\
\hline Alemanes & 10 & $10 \%$ & 10 & $13 \%$ \\
\hline Belgas & 10 & $10 \%$ & 6 & $8 \%$ \\
\hline Griegos & 8 & $8 \%$ & 5 & $6 \%$ \\
\hline Suecos & 4 & $4 \%$ & 4 & $5 \%$ \\
\hline Austríacos & 4 & $4 \%$ & 2 & $3 \%$ \\
\hline Portugueses & 3 & $3 \%$ & 2 & $3 \%$ \\
\hline Holandeses & 3 & $3 \%$ & 0 & $0 \%$ \\
\hline Total & 103 & $100 \%$ & 80 & $100 \%$ \\
\hline
\end{tabular}




\section{Bibliografía}

Allan, G. H. (1979) A sociology of friendship and kinship. London, George Allen and Unwin.

Allan, G. H. (1989) Friendship: Developping a social perspective. London, Harversten Neatsheaf.

Bidart, C. (1991) "L'amitié, les amis, leur histoire." Sociétés contemporaines, No 5.

Bidart, C. (1993) Les semblables, les amis et les autres: sociabilité et amitié, Tesis Doctoral, Marseille EHESS, 1993.

Bloch F. y Buisson M. (1991) "Du don à la dette : la construction du lien social familial." Revue du MAUSS, 11 : 54-71.

Boer, P., Huisman, M., Snijders, T.A.B., y Zeggelink, E.P.H. (2001). StOCNET: an open software system for the advanced statistical analysis of social networks. Groningen: ProGAMMA / ICS. http://stat.gamma.rug.nl

Boudon R. (1984) La place du désordre : critique des théories du changement social Sociologies, PUF, Paris.

Brewer, M.B. y Gaertner, S.L. (2001). Toward reduction of prejudice: Intergroup contact and social categorization. In Rupert Brown and Samuel L. Gaertner (eds.), Blackwell Handbook of Social Psychology: Intergroup processes. Blackwell Publishers.

Breiger, R. y Roberts, J. M. (1998) "Solidarity and social networks" en P. Doreian y T. Farraro The problem of solidarity: Theories and Models Amsterdam: Gordon and Breach, p. 239-262.

Bunt G. van de (1999) Friends by choice Amsterdam Thesis Publishers ICS series.

Cucó i Giner, J. (1995) La amistad. Perspectiva antropológica. Icaria. Institut Català d'Antropologia.

Degenne , A. y Forsé, M. (1994) Les réseaux sociaux: une analyse structurale en sociologie. Paris, A. Colin.

Derlaga, V. J. y Winstead, B, A. (1986) Friendship as social interaction, New York, Springer-Verlag.

Deutsch, K. W. (1952) "Nationalistics responses to study abroad" Archivos de la NAFSA, NAFSA 5th Annual National Conference, Michigan State University, spring 1997.

Duijn, M.A.J. van (1995). "Estimation of a random effects model for directed graphs". En T.A.B. Snijders (Ed.) SSS'95 Symposium Statistische Software, nr. 7. Toeval zit overal: programmatuur voor random coeficient modellen (113-131). Groningen: ProGAMMA.

Duijn, M.A.J. van; Zeggelink, E.; Huisman, M.;Stokman, F. N.; Wasseur, F.W. (2002) "Evolution of sociology freshmen into a friendship network" a publicar en Journal of Mathematical Sociology.

Federico, A. de (1997) L'amitié dans le programme ERASMUS : Une analyse de la création et durée des relations d'amitié des boursiers Erasmus à Lille. Tesina de doctorado bajo la dirección de Alexis Ferrand, Institut de Sociologie, Université des Sciences et Technologies de Lille (Francia). 
Federico, A. de (1998). "La movilidad estudiante en el espacio europeo: emergencia y efectos de las relaciones de amistad." Presentado en el IV Congreso Vasco de Sociología. Bilbao 26-28 Febrero 1998.

Federico, A. de (2001). "Networks and identities: Borders of solidarity and borders of identification of European exchange students". Presentado en la conferencia 'Networks And Transformations' Global Studies Association (GSA), Manchester Metropolitan University, Manchester, UK, 2-4 julio 2001. Disponible en: http://www.mmu.ac.uk/gsa/first conference.html

Federico, A. de (2002a). "Tendiendo puentes : de Lilnet a Redes. Introducción teórica a las relaciones entre micro y macro. Contribuciones actuales del análisis estructural" Número especial de REDES. Revista hispana para el análisis de redes sociales. Vol. 3, 2002. http://revista-redes.rediris.es/vol3/vol3 1.htm

Federico, A. de (2002b). "Amistad e identificación. Las micro fundaciones de las pertenencias macro. Amigos europeos e identidad europea." Número especial de REDES. Revista hispana para el análisis de redes sociales. Vol. 3, 2002. http://revista-redes.rediris.es/html-vol3/vol3 6.htm

Federico, A. de (2002c) "Introduction à SIENA pour l'analyse longitudinale de réseaux complets" presentado en el seminario GARES, CLERSÉ, Université des Sciences et Technologies, Lille (Francia) 28 de febrero de 2002. Disponible en: http://wwsympa.univ-lille1.fr/wws/arc/gares/2002-02/msg00003.html bajo el nombre "Intro_SIENA.rtf"

Feld, S. L. (1997) "Structural embeddedness and stability of interpersonal relations". Social networks, 19(1) : 91-95.

Ferrand, A. (1993) L'Analyse des réseaux personnels. Habilitation à diriger des recherches en sciences sociales et humaines auprès de I'Université de Lille.

Ferrand, A. (1997) "Influence des réseaux de confidence sur les relations sexuelles" La sexualité au temps du Sida. Paris INSERM, P.U.F, 1997.

Ferrand, A. y Mounier, L. (1999) "The diversity of personal networks in France ; social stratification and relational structures" in Wellman B. (ed.) Networks in the global village, Boulder, Westview Press, 185-224.

Fischer C.S. (1982a) To dwell among friends : personal network in town and city. Chicago, University Press.

Fischer C.S. (1982b) "What do we mean by friends ? An inductive study " Social Networks, n³.

Flap, H. y Volker, B. (2002) "Occupational community and solidarity at work" presentado en la Second European Thematic Conference for Network Analysts. "Lilnet. Micro-macro relations : advances in the contribution of structural analysis", Lille, 30-31 mayo 2002.

Frank, O. y Strauss, D. (1986) "Markov graphs." Journal of the American Statistical Association, 81, 832-842.

Godbout J. y Charbonneau J. (1993) "La dette positive dans le lien familial". Revue du MAUSS, "Ce que donner veut dire", 235-256.

Granovetter, M. S. (1973) "The strength of week ties" American Journal of Sociology, 81 : 1287-1303.

Hallinan, M. T. (1979) "The process of friendship formation", Social Netwoks, 1979, $1: 193-210$. 
Hiramatsu, H. (1999) "Longitudinal data analysis on friendship network formation of Japanese students" Sunbelt XIX International Conference on Social Networks, Charleston, 18-21 febrero 1999.

Holland, P. W. y Leinhardt, S. (1976) "Local structures in social networks" En: D. Heise (ed.), Sociological methodology. San Francisco: Jossey Bass.

Holland, P. W. y Leinhardt, S. (1981) "An exponential family of probability distributions for directed graphs (with discussion)" Journal of the American Statistical Association, 1981, 76, 33-65.

Jaccard, P. (1900) "Contribution au problème de l'immigration post-glaciaire de la flore alpine". Bulletin de la Société Vaudoise sur la Science Naturelle 37, p. 547579.

Klapisch, C. (Francia, 2002) L'auberge espagnol.

Kurth, S.B. (1970) "Friendships and friendly relations", en G.J. Mc Call, M.M. Mc Call, N. K. Denzin, G. D. Suttles, y S.B. Kurth (eds.) Social Relationships. Chicago, Aldine.

Lazarsfeld, P. y Merton, R. (1954) "Friendship as a social process", en Berger et al Freedom and control in modern society. Princeton, Van Nostrand.

Lazega, E. (2001) The Collegial Phenomenon : The Social Mechanisms of Cooperation Among Peers in a Corporate Law Partnership, Oxford, Oxford University Press.

Lazega, E. y van Duijn, M. (1997) "Position in formal structure, personal characteristics and choices of advisors in a law firm : a logistic regression model for dyadic network data." Social Networks, 19 :375-397.

Leenders, R. (1995) Structure and influence Amsterdam, Thesis Publisher.

Opper, S. Teichler, U. y Carlson, J. (1990) Impact of Study Abroad Programmes on Students and Graduates. London, Jessica Kingsley Publishers.

Pahor, M. (version de noviembre 2002) "Measures of actors activity and attractiveness". Trabajo sin publicar.

Paine R. (1969) "In search of friendship". Man, vol. 4 p. 505-524.

Perlman, D. y Fehr, B. (1986) "Theories of friendship: The analysis of Interpersonal Attraction" En Derlaga, V. J. y Winstead, B, A. Friendship as social interaction, New York, Springer-Verlag.

Pettigrew, T.F. \& Meertens, R.W. (1995). "Subtle and blatant prejudice in Western Europe". European Journal of Social Psychology, 25, 57-75.

Requena, (1994) F. Amigos y redes sociales. Madrid, Centro de Investigaciones Sociológicas, Siglo XXI, 1994

Ruiz-Gelices, E., King, R. Favell, A. (2000) "International Student Migration in Europe and the Institutionalisation of a 'European Identity'" International Migration: New Patterns, New Theories, 11-13 September, Nottingham Trent University.

Selltiz , C. Cook, S. W. "Factors influencing attitudes of a foreign students towards host country." The journal of social issues, 1962, 18 (1) 7-23.

Shild, E. O. "The foreign Student as a Stranger Learning the Norms of the Host Culture" The Journal of Social Issues, 1962, 18 (1) 41-54.

Simmel, G. (1908) "Excursus sur l'étranger" en la edición francesa Sociologie. Presses Universitaires de France, 1992, p.663-668. 
Snijders, T. A. B. (1996) "Stochastic Actor-Oriented Models for Network Change" Journal of Mathematical Sociology, 21 (1-2) : 149-172.

Snijders, T. A. B. (2001) "The statistical Evaluation of Social Network Dynamics" Sociological Methodology.

http://statt.gamma.rug.nl/snijders/Siena.html

Snijders, T. A. B. (2002) "Marco chain monte carlo estimation of exponential random graph models" Journal of Social Structure.

Snijders, T. A. B. Duijn, M. van (1997) "Simulation for statistical inference in dynamic network models" In Conte, R. Hegselmann, R. Terna, P. (Eds.) Simulating social phenomena Berlin Springer. p. 493-512.

Stroeber, W. Lenkert, A. Jonas, K. (1988) "Familiarity may breed contempt. The impact of student exchange on national sterotypes and attitudes" En Stroebe, W. Kruglanski, A.W., Bar-Tal, D. Hewstone, M. (eds.) The social Psychology of intergroup conflict: Theory, research and applications. Springer, Berlin, p. 167-187.

Suitor, J. y S. Keeton. (1997) "Once a friend, always a friend? Effects of homophily on women's support networks across a decade." Social Networks, 19 : 51-62.

Vos, H. y Wielers, R. (2002) "Calculativeness, trust, and the reciprocity complex: is the market the domain of cynism?" a publicar en B. Nooteboom y F. E. Six, The trust process within organizations: empirical studies of the determinants and the process of trust development. Cheltenham, UK: Edward Elgar.

Wasserman, S. Faust K. (1994) Social Network Analysis: Methods and Applications Cambridge, Cambridge University Press.

Wasserman, S. y Pattison, P. (1996) "Logit models and logistic regression for social networks : I. An introduction to Markov graphs and $p^{*}$." Psychometrika, 61, 401425.

Wellman, B. Carrington, P. J. and Hall, A. (1988) "Network as personal communities" en Wellman, B. Berkowitz Social Structures: A Network Approach, Cambridge, University Press, p130-84.

Wellman, B. Wortley, S. (1990) "Different Strokes from different folks: Community ties and social support: Wich ties provide what kinds of social support." American Jourrnal of sociology, Novembre, 96 (3) : 558-88.

Williamson, O. E. (1993) "Calculativeness, trust and economic organization" Journal of Law and Economics, 36, 453-486.

Zeggelink, E. (1993) Strangers into friends. The evolution of friendship networks. Amsterdam, Thesis Publisher.

Zeggelink, E. P. H. ; van Duijn, M. ; Hiramatsu, H. ; Stockman, F. N. ; van Oosten, R. ; Wasseur, F. (1999) "Determinants of friendship formation : a comparative analysis of firendship formation among freshmen in Japan and the Netherlands" Sunbelt XIX International Conference on Social Networks, Charleston, 18-21 febrero 1999.

Zeggelink, E. P. H. ; Hiramatsu, H. ; Stockman, F. N. ; van Duijn, M. ; Wasseur, F. (1997) "Friendship formation over time among sociology students : a comparison between Japan and the Netherlands" XVIII Conferencia Internacional y V Conferencia Europea de Análisis de Redes Sociales. Sitges 27-31 Mayo 1997. 